\title{
Vacuum stability of asymptotically safe two Higgs doublet models
}

\author{
Peter Schuh ${ }^{\mathrm{a}}$ \\ Fakultät Physik, TU Dortmund, Otto-Hahn-Str. 4, 44221 Dortmund, Germany
}

Received: 14 November 2018 / Accepted: 25 October 2019 / Published online: 11 November 2019

(C) The Author(s) 2019

\begin{abstract}
We study different types of Two Higgs Doublet Models (2HDMs) under the assumption that all quartic couplings' beta functions vanish simultaneously at the Planck scale. The Standard Model seems to display this property almost accidentally, because the Higgs boson mass is close to $125 \mathrm{GeV}$. This also ties closely into the question of whether the theory is stable or metastable. We investigate if such "fixed points" can exist in various $\mathbb{Z}_{2}$-symmetric $2 \mathrm{HDM}$ subclasses, and if the theories that meet these conditions are phenomenologically viable, as well as vacuum stable. We find that the fixed point condition drastically reduces the parameter space of $2 \mathrm{HDM}$ theories, but can be met. Fixed points can only exist in type II and type $\mathrm{Y}$ models, in regions of large $\tan \beta$, and they are only compatible with all existing experimental bounds if the $\mathbb{Z}_{2}$-symmetry is at least softly broken, with a soft breaking parameter of at least $M_{12}>70 \mathrm{GeV}$ $(380 \mathrm{GeV}$ ) for type Y (type II) models. The allowed region falls into the alignment limit, with the mixing angle combination $|\alpha-\beta| \approx \frac{\pi}{2}$. While there are both vacuum-stable and vacuum-unstable solutions, only the vacuum-unstable ones really agree with Standard-Model-like CP-even Higgs boson mass values of $125 \mathrm{GeV}$. The vacuum-stable solutions favour slightly higher values. While scenarios of asymptotically safe 2HDM exist, they cannot improve over the Standard Model regarding the question of vacuum stability.
\end{abstract}

\section{Contents}

1 Introduction $\ldots \ldots \ldots \ldots \ldots \ldots$

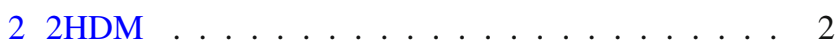

2.1 General properties of the 2HDM . . . . . . 2

2.2 Vacuum stability ............. . . 3

2.3 Limits on 2HDM parameter space . . . . . . . 4

3 Solving the fixed point equations . . . . . . . . 4

4 The CP-conserving $2 \mathrm{HDM}$ with $\mathbb{Z}_{2}$ symmetry . . . 5

a e-mail: peter.schuh@udo.edu
4.1 Higgs boson mass spectra . . . . . . . . . . 6

4.2 Vacuum stability of the fixed point solutions . . 7

5 The 2 HDM with softly broken $\mathbb{Z}_{2} \ldots \ldots \ldots$

5.1 Stability analysis . . . . . . . . . . . 9

5.2 Uncertainty estimates . . . . . . . . . . . . 9 9

6 Summary . . . . . . . . . . . . . . . 11

Appendix: $\beta$ functions . . . . . . . . . . . 13

References . . . . . . . . . . . . . . 15

\section{Introduction}

The discovery and the mass measurement of a Standard Model (SM)-like Higgs boson by ATLAS and CMS in 2012 $[1,2]$ so far rank among the most impactful events in this century's particle physics. It is an interesting situation that the Higgs mass of $m_{H}=(125.5 \pm 0.5) \mathrm{GeV}$ lies right at the edge of the so-called stability bound [3-5]. Extrapolating from the Higgs mass value to very short distances shows that the LHC result seems to hint at a quartic coupling of $\lambda=0$ at Planck scale-like energies, and similarly the renormalisation group (RG) beta function tends to $\beta_{\lambda}\left(m_{P l}\right) \sim 0$.

The argument also works in reverse: Before the LHC experiments had discovered a Higgs boson, calculations were performed to show that initial conditions of $\lambda=0$ and $\beta_{\lambda}=0$ at high scales naturally point to Higgs mass values around $125 \mathrm{GeV}$ [6], as do the combination of a vanishing beta function at high scales and the experimental measurement of the top quark mass, or of a vanishing quartic coupling at high scales and the top quark mass [7]. The idea of vanishing beta functions suggests a link to the field of Asymptotic Safety [8-10], in which RG flow fixed points play a critical role. Originally an approach to quantum gravity, Asymptotic Safety immediately ensures that theories remain valid up to highest scales. It has in recent years become a point of interest in SM extensions, as a mechanism for UV completion or generalised renormalisability 
[11-16]. Most notably, recent works have emphasized that the interplay of Yukawa interactions with Higgs-like scalar fields is one of the key aspects in understanding Asymptotic Safety [17].

The relation between vanishing quartic couplings and vanishing beta functions at high scales and the measured Higgs mass at the LHC may be coincidental. On the other hand, the question of vacuum stability remains. Experimental results suggest that the SM vacuum is metastable, although agreement on how strong a statement can be made has not yet been reached [18]. Here, we study how models with an enlarged scalar sector behave in this regard. Two Higgs Doublet Models (2HDMs) are arguably among the most minimal and natural extensions of the SM, but nevertheless possess immense phenomenological flexibility and offer avenues to explore common open questions, such as baryon asymmetry [19-21] or dark matter candidates [22-24], among others. At the same time, 2HDMs fit well with the experimental Higgs measurements collected so far [25-27]. On the other hand however, the parameter space introduced by the $2 \mathrm{HDM}$ is quite vast, and somewhat complex. To this end, we look at 2HDMs and investigate if they can support the Asymptotic Safety scenario in a way similar to the SM. Specifically, we examine 2HDMs that exhibit simultaneously vanishing quartic coupling beta functions $\beta_{\lambda_{i}}(\mu)$ at the Planck scale $m_{P l}=1.2 \cdot 10^{19} \mathrm{GeV}$. As such, the concept of Asymptotic Safety will function first as a goal in this work, as it is a fundamentally interesting question which theories exhibit UV fixed points under which circumstances. The second step however, is to utilise Asymptotic Safety as a tool: As these fixed point conditions are strong demands in a model with an extended scalar sector, that means that if fixed points can be found, demanding the presence of Asymptotic Safety then becomes a powerful lever to significantly reduce the possible open parameter space of the general $2 \mathrm{HDM}$. If phenomenologically viable asymptotically safe $2 \mathrm{HDM}$ can be found, we then ask if they can improve on the SM in the question of vacuum stability.

This paper is organized as follows: In Sect. 2, general properties of $2 \mathrm{HDMs}$ are reviewed. A detailed outline of how the analyses are performed are then given in Sect. 3. Sections 4 and 5 subsequently treat different types of $2 \mathrm{HDMs}$, including the complete softly-broken $\mathbb{Z}_{2-}$ symmetric model. An Appendix contains the complete twoloop beta functions of all 2HDM couplings used in this work.

\section{2HDM}

In this section, we briefly review the features of the general 2HDM, before reviewing the current state of bounds on the model from different sources.

\subsection{General properties of the 2HDM}

A 2 HDM contains two SU(2) doublets $\Phi_{1}, \Phi_{2}$ [28]. The most general scalar potential takes the form:

$$
\begin{aligned}
V= & m_{11}^{2} \Phi_{1}^{\dagger} \Phi_{1}+m_{22}^{2} \Phi_{2}^{\dagger} \Phi_{2}+\left(M_{12}^{2} \Phi_{1}^{\dagger} \Phi_{2}+\text { h.c. }\right) \\
& +\frac{1}{2} \lambda_{1}\left(\Phi_{1}^{\dagger} \Phi_{1}\right)^{2}+\frac{1}{2} \lambda_{2}\left(\Phi_{2}^{\dagger} \Phi_{2}\right)^{2} \\
& +\lambda_{3}\left(\Phi_{1}^{\dagger} \Phi_{1}\right)\left(\Phi_{2}^{\dagger} \Phi_{2}\right)+\lambda_{4}\left(\Phi_{1}^{\dagger} \Phi_{2}\right)\left(\Phi_{2}^{\dagger} \Phi_{1}\right) \\
& +\left[\frac{1}{2} \lambda_{5}\left(\Phi_{1}^{\dagger} \Phi_{2}\right)^{2}+\lambda_{6}\left(\Phi_{1}^{\dagger} \Phi_{1}\right)\left(\Phi_{1}^{\dagger} \Phi_{2}\right)\right. \\
& \left.+\lambda_{7}\left(\Phi_{2}^{\dagger} \Phi_{2}\right)\left(\Phi_{2}^{\dagger} \Phi_{1}\right)+\text { h.c. }\right] .
\end{aligned}
$$

In this notation, following [29], $m_{11}, m_{22}$, and $\lambda_{1}$ to $\lambda_{4}$ are real-valued, whereas $M_{12}, \lambda_{5}, \lambda_{6}$, and $\lambda_{7}$ are complex parameters. Of these 14 degrees of freedom, only eleven are physical. The rest can be absorbed by making use of the freedom of choice of bases for the SU(2) doublets $\Phi_{i}$.

For spontaneous symmetry breaking (SSB), both fields $\Phi_{1}$ and $\Phi_{2}$ are assigned a vacuum expectation value (VEV): $\left\langle\Phi_{i}\right\rangle_{0}=\left(0, \frac{v_{i}}{\sqrt{2}}\right)^{\top}$, with $v_{i}$ related to the SM VEV $v \simeq 246$ $\mathrm{GeV}$ via

$v_{1}^{2}+v_{2}^{2}=v^{2}$

The SU(2) doublets contain eight physical fields $\Phi_{i}=$ $\left(\phi_{i}^{+}, \frac{\left(v_{i}+\rho_{i}+i \eta_{i}\right)}{\sqrt{2}}\right)^{\top}$, three of which are absorbed during SSB. The remaining physical Higgs bosons after rotating into mass eigenstates are the charged Higgs $H^{ \pm}$, a pseudoscalar Higgs $A$ and two CP-even scalar Higgs $h, H$. The rotation angle diagonalising the $\mathrm{CP}$-even scalar mass matrix is conventionally called $\alpha$, the angle diagonalising the charged and CP-odd bosons is called $\beta$. The latter angle $\beta$ also appears in the ratio of $\frac{v_{2}}{v_{1}} \equiv \tan \beta$.

In general, 2HDMs permit tree-level FCNCs. According to the Paschos-Glashow-Weinberg theorem [30,31], a necessary and sufficient condition for their absence is to have all fermions of the same charge and helicity couple to the same Higgs doublet. There are effectively only four different ways of distributing fermions to doublets, as $\Phi_{1}$ and $\Phi_{2}$ are inherently interchangeable: A model in which all fermions couple to the same Higgs doublet (usually $\Phi_{2}$ ) is called type $I$, a model where up-type quarks couple to $\Phi_{2}$ and down-type quarks couple to $\Phi_{1}$ is called type II. Aligning the leptons with up-type instead of down-type quarks results in the so-called lepton-specific and flipped models, or type $X$ and type $Y$, respectively. In practice, the different types are usually enforced through discrete $\mathbb{Z}_{2}$-symmetries. The exact charge assignments of Higgs and fermion fields are listed in Table 1 [32]. For our purpose the leptons only contribute minor corrections when compared to the quarks, 
Table $1 \mathrm{Z}_{2}$ charge assignments of Higgs doublets and fermion fields for different $2 \mathrm{HDM}$ types

\begin{tabular}{llllrrr}
\hline & $\Phi_{1}$ & $\Phi_{2}$ & $u^{R}$ & $d^{R}$ & $l^{R}$ & $Q^{l}, L^{l}$ \\
\hline Type I & -1 & 1 & 1 & 1 & 1 & 1 \\
Type II & -1 & 1 & 1 & -1 & -1 & 1 \\
Type X & -1 & 1 & 1 & 1 & -1 & 1 \\
Type Y & -1 & 1 & 1 & -1 & 1 & 1 \\
\hline
\end{tabular}

so the primary computational focus will be on type I and type II models.

The Yukawa Lagrangian for type I and type II 2HDMs are hence given by:

$$
\begin{aligned}
-\mathcal{L}_{Y}^{I}= & \left(\bar{Q}_{i}^{l} \Phi^{2} Y_{i j}^{d} d_{j}^{R}+\bar{Q}_{i}^{l} \tilde{\Phi}^{2} Y_{i j}^{u} u_{j}^{R}\right. \\
& \left.+\bar{L}_{i}^{l} \Phi^{2} Y_{i j}^{l} l_{j}^{R}\right)+h . c ., \\
-\mathcal{L}_{Y}^{I I}= & \left(\bar{Q}_{i}^{l} \Phi^{2} Y_{i j}^{d} d_{j}^{R}+\bar{Q}_{i}^{l} \tilde{\Phi}^{1} Y_{i j}^{u} u_{j}^{R}\right. \\
& \left.+\bar{L}_{i}^{l} \Phi^{1} Y_{i j}^{l} l_{j}^{R}\right)+ \text { h.c. },
\end{aligned}
$$

where $Y^{u, d, l}$ are the Yukawa matrices for up-, down-, and lepton type particles, $Q^{l}, L^{l}, u^{R}, d^{R}$, and $l^{R}$ are left- and righthanded quark and lepton fields respectively, and $i, j$ denote the generations in flavour space. In our calculations, only the dominant $y_{33}$ entries generated by the top quark, the bottom quark, and the tau lepton respectively, will be considered. Thus, the Yukawa matrices are assumed to have the simplified structures $Y^{u}=\operatorname{diag}\left(0,0, \lambda_{t}\right), Y^{d}=\operatorname{diag}\left(0,0, \lambda_{b}\right)$ and $Y^{l}=\operatorname{diag}\left(0,0, \lambda_{\tau}\right)$.

Under the $\Phi_{1} \rightarrow-\Phi_{1} \mathbb{Z}_{2}$ symmetry mentioned above, it follows that $\lambda_{6}=\lambda_{7}=0$, which leads to a mass matrix for the $\mathrm{CP}$-even neutral scalars of the form:

$M_{h / H}^{2}=\left(\begin{array}{cc}m_{11}^{2}+\frac{3}{2} \lambda_{1} v_{1}^{2}+\frac{3}{2} \lambda_{345} v_{2}^{2} & -\operatorname{Re}\left(M_{12}^{2}\right)+\lambda_{345} v_{1} v_{2} \\ -\operatorname{Re}\left(M_{12}^{2}\right)+\lambda_{345} v_{1} v_{2} & m_{22}^{2}+\frac{3}{2} \lambda_{2} v_{2}^{2}+\frac{3}{2} \lambda_{345} v_{2}^{2}\end{array}\right)$,

with $\lambda_{345}=\lambda_{3}+\lambda_{4}+\operatorname{Re}\left(\lambda_{5}\right)$. The terms $m_{11}$ and $m_{22}$ can be eliminated using the minimum conditions from SSB, that is $\frac{\partial V}{\partial v_{i}}=0$ :

$$
\begin{aligned}
& m_{11}^{2} v_{1}-\operatorname{Re}\left(M_{12}^{2}\right) v_{2}+\frac{\lambda_{1}}{2} v_{1}^{3}+\frac{\lambda_{345}}{2} v_{1} v_{2}^{2}=0, \\
& m_{22}^{2} v_{2}-\operatorname{Re}\left(M_{12}^{2}\right) v_{1}+\frac{\lambda_{2}}{2} v_{2}^{3}+\frac{\lambda_{345}}{2} v_{1}^{2} v_{2}=0 .
\end{aligned}
$$

The charged and the pseudoscalar Higgs mass matrices are given by:

$$
\begin{aligned}
M_{H^{ \pm}}^{2} & =\frac{v^{2}}{v_{1} v_{2}}\left(\operatorname{Re}\left(M_{12}^{2}\right)-\frac{\lambda_{4}+\operatorname{Re}\left(\lambda_{5}\right)}{2} v_{1} v_{2}\right)\left(\begin{array}{cc}
\frac{v_{2}}{v_{1}} & -1 \\
-1 & \frac{v_{1}}{v_{2}}
\end{array}\right), \\
M_{A}^{2} & =\left(\operatorname{Re}\left(M_{12}^{2}\right)-\operatorname{Re}\left(\lambda_{5}\right) v_{1} v_{2}\right)\left(\begin{array}{cc}
\frac{v_{2}}{v_{1}} & -1 \\
-1 & \frac{v_{1}}{v_{2}}
\end{array}\right) .
\end{aligned}
$$

Both have one zero eigenvalue, corresponding to the charged and the pseudoscalar Goldstone boson, respectively. The pseudoscalar mass vanishes for $M_{12}=\lambda_{5}=0$, because of an additional accidental spontaneously broken $\mathrm{U}(1)$-symmetry.

\subsection{Vacuum stability}

In 2HDMs, to be vacuum-stable the potential needs to be bounded from below in all directions. This is the case if and only if the following set of inequalities is met [22]:

$$
\begin{aligned}
\lambda_{1} & >0, & \lambda_{2} & >0, \\
\lambda_{3}+\sqrt{\lambda_{1} \lambda_{2}} & >0, & \lambda_{3}+\lambda_{4}+\sqrt{\lambda_{1} \lambda_{2}} & >\left|\lambda_{5}\right| .
\end{aligned}
$$

For absolute stability, we require the conditions (2.9) to be fulfilled at all scales up to $m_{P l}$. For ease of language, we will usually refer to a potential as stable, if it exhibits absolute stability, and as unstable otherwise. As will be discussed in more detail in Sect. 4.2, a number of models discussed in this paper exhibit very similar behaviour to the SM, in that a quartic coupling becomes negative, but not very negative, at high scales. To decide if these models are in fact truly unstable or only metastable, we calculate the tunnelling rate to the lower vacuum, and make an estimate on the vacuum lifetime. Unlike the SM, theories with more than one Higgs doublet can display a range of different vacuum configurations [33]: Not only can there be more than one minimum at the same time, but the minima can also be of $\mathrm{CP}$ breaking type, when the VEVs have a relative complex phase, or of charge breaking type, with one VEV carrying an electric charge. It has however been shown that minima of different types (i.e. CPbreaking, charge-breaking, or normal) cannot exist simultaneously within the same model [34-36]. By requiring the model to fulfil the minimum conditions for normal-type minima given by Eqs. (2.5) and (2.6), it is therefore assured that the absolute minimum of the theory is also normal. Nevertheless, the possible existence of potentially deeper minima means that even a potential bounded from below could just be a false vacuum state, and lie at risk to decay into the global vacuum. In this scenario, usually referred to as panic vacuum, it once again becomes a relevant question if the tunnelling time between the minimal is large compared to the age of the universe. A recent detailed treatment of the relations between different neutral 2HDM minima and the lifetimes of false vacua can be found in [33]. To check if the minimum at $v=246 \mathrm{GeV}$ is global, true minimum of the 
potential, the following necessary and sufficient condition has been established in [37]:

$M_{12}^{2}\left(m_{11}^{2}-\sqrt{\frac{\lambda_{1}}{\lambda_{2}}} m_{22}^{2}\right)\left(\tan \beta-\sqrt[4]{\frac{\lambda_{1}}{\lambda_{2}}}\right)>0$.

The authors in [33] also consider whether transitions are possible between degenerate vacuum states, i.e. solutions of the form $\left\{-v_{1},-v_{2}\right\}$. We leave that possibility aside in this work.

\subsection{Limits on 2HDM parameter space}

While the 2HDM is a relatively simple SM extension, it still contains up to eleven new free parameters (six in the type II models studied below). On the other hand, the model's high popularity means that its parameter space has been comprehensively explored and constrained from both the theoretical and the experimental side, and in particular by recent LHC data [38-40]. At this point, we briefly review current bounds, more thorough discussions of different aspects can be found for example in [41-47].

In essence, constraints on the 2HDM parameter space can be sorted into three categories: Theory bounds are generated by requiring the model to possess certain features, commonly referred to as positivity (the Higgs potential must be bounded from below, cf. Eq. (2.9)), perturbativity (quartic couplings must not be large), and unitarity (of the S-matrix of $2 \rightarrow 2$ scattering amplitudes) [48,49]. Secondly, there are mass bounds on the physical Higgs bosons from signal strength data by the ATLAS and CMS collaborations. These searches have confirmed the existence of a $125 \mathrm{GeV} \mathrm{CP-even} \mathrm{scalar} \mathrm{eigenstate,}$ and they also show that this boson couples to vector bosons and fermions in a very SM-Higgs-like fashion [50-53]. Furthermore, the absence of heavier resonances so far translates to mass bounds for the other Higgs eigenstates. Lastly, there are implications for the 2HDM from flavour physics [54]. Most notably, $\mathcal{B}(b \rightarrow s \gamma)$ measurements exclude charged Higgs masses smaller than $m_{H^{+}}=580 \mathrm{GeV}$ [55] in type II/type Y models, lower bounds on $\tan \beta$ can be extracted from $B_{s}$ mass differences and leptonic decays [56].

Together, these bounds can be combined to make a number of statements: The masses of the three additional Higgs bosons all must be large, the mass differences between them, however, small. The rotation angles must fulfil $|\beta-\alpha| \approx \frac{\pi}{2}$, ensuring that the mass basis of the CP-even scalar states aligns with the SM gauge eigenbasis. These features are thus usually referred to as alignment limit [57-60]. It should be noted that some studies have used fine-tuning arguments to impose stronger bounds on $\tan \beta$, and successively to the heavy Higgs boson masses [42]. Since the RG methods employed in this work contain a certain degree of tuning by design, they offer an alternative as to why these large $\tan \beta$ regions may yet be phenomenologically viable. As a consequence, our mass bounds are slightly more conservative than some.

\section{Solving the fixed point equations}

We pursue the question whether 2HDMs support "fixed points" at the Planck scale in the same way the SM does, and if the resulting models are vacuum-stable. The fixed point condition reads:

$\beta_{\lambda_{i}}\left(m_{P l}\right)=0 \quad \forall i$,

i.e., the beta functions of all quartic couplings $\lambda_{i}$ present in the scalar potential are to have a root at the Planck mass $m_{P l}$. Because of contributions from gauge and Yukawa couplings, the condition of $\beta_{\lambda_{i}}=0$ is not technically sufficient to define a fixed point, nor does it necessarily lead to an asymptotically safe theory by itself; for recent progress in BSM model building see e.g. [11]. Still, because of similarities to the SM case and for convenience, the terms fixed point and fixed point condition will be used in this context, effectively interpreting effects disturbing the equilibrium into the realm of beyond the Planck scale physics.

While in the SM there is only one quartic Higgs coupling $\lambda$, the $2 \mathrm{HDM}$ potential with a $\mathbb{Z}_{2}$ symmetry protecting flavour conservation can contain up to five quartic terms (one of which may be complex). This means that compared to the SM, the fixed point condition has a much higher impact in terms of limiting the parameter space of the theory.

The search for fixed points comes down to solving the system of differential equations given by the beta functions of the running couplings of the theory. It involves the gauge couplings $g_{1}, g_{2}, g_{3}$, the quartic scalar couplings $\lambda_{i}$ and the Yukawa couplings $\lambda_{t}, \lambda_{b}, \lambda_{\tau}$. The complete two-loop expressions for the most general beta functions used are calculated with the Mathematica package SARAH [61,62], and listed in Appendix A. Since the coefficients $m_{11}$ and $m_{22}$ of the dimension-two-operators do not appear directly in the beta functions of any other couplings, $m_{i i}$ can be ignored at this point and determined with help of the minimum conditions at the electroweak scale, see Eqs. (2.5) and (2.6). The soft breaking parameter $M_{12}$ also does not appear in the beta functions of quartic, gauge, or Yukawa couplings, and will be treated as a free parameter.

While the quartic couplings are already fixed implicitly by (3.1), the remaining initial conditions are given explicitly at low scales: Both gauge couplings and Yukawa couplings can be determined from experimental measurements. The $\overline{\mathrm{MS}}$ gauge coupling initial conditions for $g_{1}$ and $g_{2}$ are calculated using the fine structure constant $\alpha^{-1}\left(M_{Z}\right)=127.95 \pm 0.017$ and the weak mixing angle $\sin ^{2} \theta_{W}=0.23129 \pm 5 \cdot 10^{-5}$ $[63,64]$ to: 
$g_{1}\left(M_{Z}\right)=0.35, \quad g_{2}\left(M_{Z}\right)=0.65, \quad g_{3}\left(M_{Z}\right)=1.2$.

Uncertainties on gauge coupling initial values, including the strong coupling, are small enough to be inconsequential. The relations between Yukawa couplings and quark masses are model-dependent. In a type II model, the Yukawa couplings are related to the quark masses by the tree-level relations:

$\lambda_{t}\left(m_{t}\right)=\frac{\sqrt{2} m_{t}}{v_{2}}, \quad \lambda_{b}\left(m_{b}\right)=\frac{\sqrt{2} m_{b}}{v_{1}}, \quad \lambda_{\tau}\left(m_{\tau}\right)=\frac{\sqrt{2} m_{\tau}}{v_{1}}$,

while in the type I model the bottom quark Yukawa coupling is instead determined by the other VEV: $\lambda_{b}\left(m_{b}\right)=\frac{\sqrt{2} m_{b}}{v_{2}}$. The $\overline{M S}$ quark masses used are $m_{b}\left(m_{b}\right)=4.18 \pm 0.03 \mathrm{GeV}$ and $m_{t}\left(m_{t}\right)=160_{-4.3}^{+4.8} \mathrm{GeV}$, the $\tau$-lepton mass is given by $m_{\tau}\left(m_{\tau}\right)=1.78 \mathrm{GeV}$ [64]. For the purpose of this paper, it is assumed that all non-SM effects only affect the running from the electroweak scale onwards. In other words, the Yukawa couplings are run up to $M_{Z}$ under SM-like conditions, at which point the $\tan \beta$-enhancement is switched on. The effective initial values used are thus:

$\lambda_{t}\left(M_{Z}\right)=\frac{0.95}{\sin \beta}, \quad \lambda_{b}\left(M_{Z}\right)=\frac{0.176}{\cos \beta}, \quad \lambda_{\tau}\left(M_{Z}\right)=\frac{0.98}{\cos \beta}$.

The so defined initial value problem is solved numerically.

With the full RG flow of all couplings known, their low scale values are used to determine the mass spectrum of the theory using the matrices given in Sect. 2. The results depend on the parameters treated as free (in these cases $\tan \beta$ and later $M_{12}$ ) and on the experimentally determined coupling initial conditions, but beyond this are a direct consequence of the theory itself and the fixed point assumption.

With all couplings known at all scales, the question of vacuum stability can also be answered: For a solution to be absolutely vacuum-stable, the quartic couplings must fulfil the inequalities given by Eq. $((2.9))$ at all energy scales up to $\mu=m_{P l}$.

\section{The CP-conserving 2HDM with $\mathbb{Z}_{2}$ symmetry}

We study a $2 \mathrm{HDM}$ with a discrete $\mathbb{Z}_{2}$ symmetry $\left(\Phi_{1} \rightarrow\right.$ $-\Phi_{1}, \Phi_{2} \rightarrow \Phi_{2}$ ) introduced in order to ensure CPconservation in the scalar sector. The scalar potential reads:

$$
\begin{aligned}
V= & m_{11}^{2} \Phi_{1}^{\dagger} \Phi_{1}+m_{22}^{2} \Phi_{2}^{\dagger} \Phi_{2} \\
& +\frac{1}{2} \lambda_{1}\left(\Phi_{1}^{\dagger} \Phi_{1}\right)^{2}+\frac{1}{2} \lambda_{2}\left(\Phi_{2}^{\dagger} \Phi_{2}\right)^{2} \\
& +\lambda_{3}\left(\Phi_{1}^{\dagger} \Phi_{1}\right)\left(\Phi_{2}^{\dagger} \Phi_{2}\right)+\lambda_{4}\left(\Phi_{1}^{\dagger} \Phi_{2}\right)\left(\Phi_{2}^{\dagger} \Phi_{1}\right) \\
& +\frac{1}{2} \lambda_{5}\left[\left(\Phi_{1}^{\dagger} \Phi_{2}\right)^{2}+\left(\Phi_{2}^{\dagger} \Phi_{1}\right)^{2}\right],
\end{aligned}
$$

with real-valued mass parameters $m_{i i}$ and quartic couplings $\lambda_{i}$. The restriction $\operatorname{Im}\left(\lambda_{5}\right)=0$ does not follow immediately from the $\mathbb{Z}_{2}$ symmetry, but can be assumed in this case without loss of generality due to the structure of the beta functions: $\lambda_{5}$ always appears in the other quartic couplings' beta functions in the form of the norm squared, $\left|\lambda_{5}\right|^{2}$, and the function $\beta_{\lambda_{5}}$ is proportional to $\lambda_{5}$, with the remainder being comprised by real-valued terms only. It follows that the function $\beta_{\lambda_{5}}$ must have a constant phase. It is also easy to see that in these circumstances for every function $\lambda_{5}(\mu)$ that solves the system of differential equations, a phase-shifted $e^{i \theta} \cdot \lambda_{5}(\mu)$ is also a solution for every constant phase $\theta$. Therefore, it suffices to look at the real values for $\lambda_{5}$ when searching for fixed points.

The different Yukawa Lagrangians in type I and type II models lead to differences in the beta functions, as the Yukawa terms will appear in the running of different couplings. As an example, in the type I model one-loop beta function of quartic coupling $\lambda_{1}$ can be written as:

$$
\begin{aligned}
\beta_{\lambda_{1}}^{1 l}= & \frac{1}{64 \pi^{2}}\left(6 g_{1}^{2} g_{2}^{2}+\left(3 g_{2}{ }^{2}-6 \lambda_{1}\right)^{2}+3\left(g_{1}{ }^{2}-2 \lambda_{1}\right)^{2}\right. \\
& \left.+8\left(6 \lambda_{1}^{2}+\lambda_{3}^{2}+\left(\lambda_{3}+\lambda_{4}\right)^{2}+\lambda_{5}^{2}\right)\right)
\end{aligned}
$$

The right hand side is expressed as a sum of perfect squares. Furthermore, it does not contain any Yukawa terms, as in type I models, $\Phi_{1}$ does not couple to any fermions. As was also discussed in [17], Yukawa couplings are often times indispensable for enabling fixed points, as they can be the only terms with a negative sign in the beta functions. The consequence of this is that the $\beta_{\lambda_{1}}^{1 l}$ above will never vanish, and type I models are excluded as viable candidates, when looking for $2 \mathrm{HDM}$ with fixed points. As a corollary, models like the one discussed in [65] extending the SM by a singlet can also not support fixed points in their new quartic coupling.

The full two-loop beta functions for the type II $\mathbb{Z}_{2}$ symmetric 2HDM are given in Appendix A. In this model, $\tan \beta$ is a free parameter that fixes the exact starting conditions. For fixed points to exist, Yukawa contributions to both Higgs field have to be big enough. This translates into a lower bound on $\tan \beta$, as the bottom-type Yukawa couplings in a type II model are $(\sin \beta)^{-1}$-enhanced compared to the SM [cf. Eq. (4.2)]. On the other hand, $\tan \beta$ cannot be too large either, or the running Yukawa couplings will become divergent below the Planck scale. Accordingly, there is a $\tan \beta$-interval in which fixed points can exist. In this two-loop framework, fixed points can be found for:

$\tan \beta \in[60,70]$.

The beta functions being of polynomial form, there is usually a finite set of fixed point solutions. We find that there are 


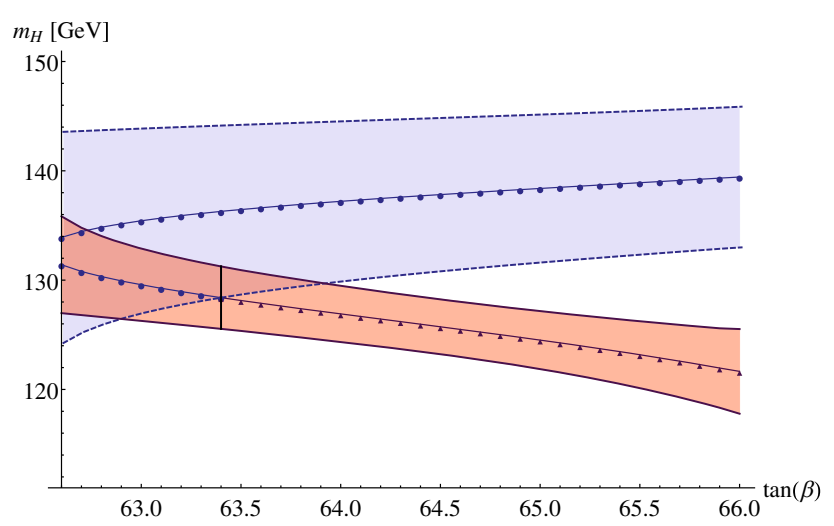

Fig. 1 Mass eigenstates of the heavier CP-even neutral scalar Higgs boson $H$ in the type II $\mathbb{Z}_{2}$-symmetric model. Vacuum-stable solutions are shown as blue circles with dashed borders, vacuum-unstable solutions as violet triangles, with the black vertical line marking the transition point. The uncertainty estimate is given by the difference between results for calculating the running of all couplings at one-loop and twoloop level, respectively

two branches: One branch is vacuum-stable for all values of $\tan \beta$ over all scales, the other one breaks the condition $\lambda_{2}>0$ at higher scales for certain values of $\tan \beta$, rendering the corresponding potential unbounded from below. For simplicity, we will in this work refer to the two branches as the vacuumstable and the vacuum-unstable one, respectively. Before we take a closer look at the exact nature of the vacua in Sect. 4.2, the values of $\lambda_{i}$ can be translated into a spectrum of masses of the physical Higgs bosons using Eqs. (2.4)-(2.7).

\subsection{Higgs boson mass spectra}

The masses for all three physical bosons in this model are shown in Figs. 1, 2 and 3, with the vacuum-stable cases shown as blue circles, and the vacuum-unstable solutions marked with purple triangles. The critical $\tan \beta$ value for stability in the lower branch is marked by a black vertical line. For $\tan \beta$ values greater than this, $\lambda_{2}$ in the unstable branch will not stay positive at all scales up to the fixed point. An estimate for the theoretical uncertainty is given by the differences between performing the RG evolution of all couplings at one-loop or at two-loop level, before determining the masses using Eqs. (2.4)-(2.7).

It becomes quickly evident that the mass spectrum produced is not in line with experimental bounds. The CP-even neutral scalar mass eigenstates are split apart wide due to the large parameter $\tan \beta$ : without significant mixing, one of the eigenstates is of the order of $v_{1}$, the other of the order $v_{2}$. The heavier CP-even eigenstate takes on mass values of around $125-130 \mathrm{GeV}$ for the (mostly) vacuum-unstable branch, and $135-140 \mathrm{GeV}$ for the stable one.The lighter CPeven eigenstate lies in the $\mathrm{O}(1) \mathrm{GeV}$ region, which is ruled out experimentally. This will be addressed in Sect. 5 .

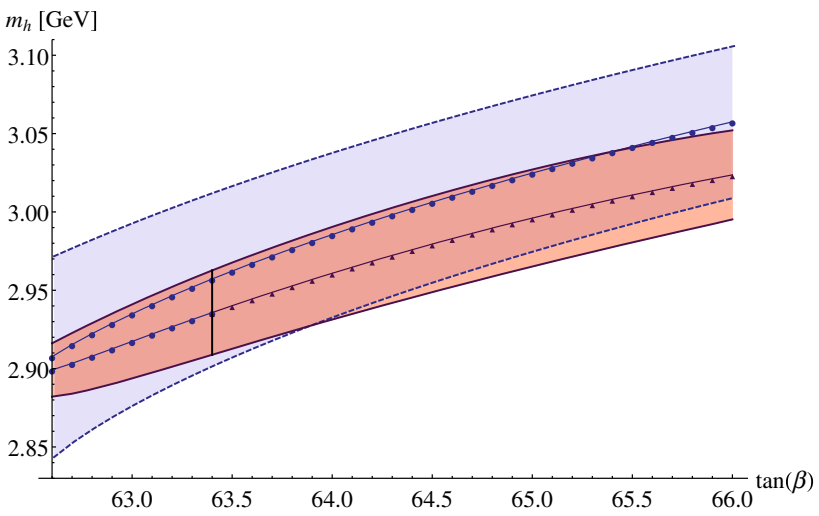

Fig. 2 Mass eigenstates of the light CP-even neutral scalar Higgs boson $h$ in the type II $\mathbb{Z}_{2}$-symmetric model, as labelled in Fig. 1

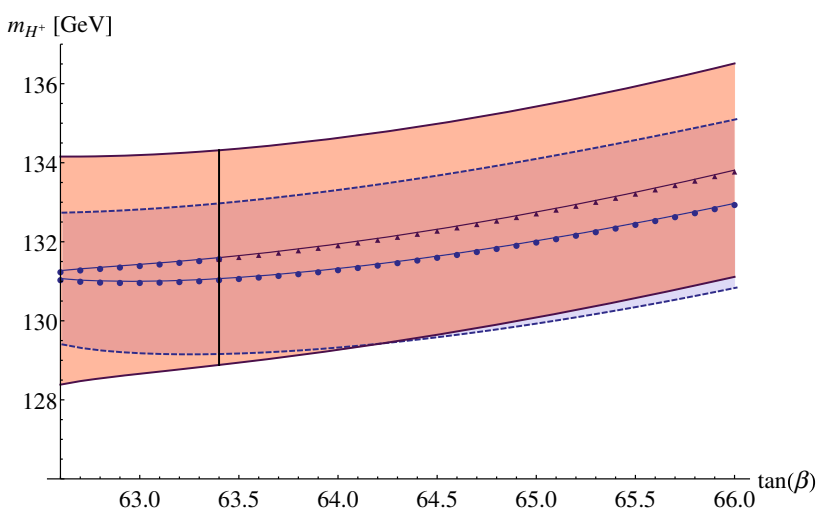

Fig. 3 Mass eigenstates of the charged Higgs boson $\mathrm{H}^{+}$in the type II $\mathbb{Z}_{2}$-symmetric model, as labelled in Fig. 1

The heavier CP-even eigenstate in the vacuum-unstable case has roughly the correct mass to be considered as a candidate for a SM-like Higgs. However, while it is theoretically possible that the observed Higgs boson at $125 \mathrm{GeV}$ is the heavier of the two $\mathrm{CP}$-even eigenstates, this configuration is heavily disfavoured by experimental observations, due to strong bounds from the $H \rightarrow h h$ decay [66]. It is therefore usually assumed in $2 \mathrm{HDMs}$ that the $125 \mathrm{GeV}$ Higgs is the lighter of the two $\mathrm{CP}$-even neutral eigenstates.

The charged Higgs boson mass is below $200 \mathrm{GeV}$, which falls into the regions excluded by $\bar{B} \rightarrow X_{s} \gamma$ measurements, as mentioned in Sect. 2.3. The pseudoscalar mass is not shown, because it vanishes completely: As it turns out, all fixed point solutions contain $\lambda_{5} \equiv 0$. In this case (with $M_{12}$ forbidden by the $\mathbb{Z}_{2}$-symmetry), this means that the model displays an accidental U(1)-symmetry which forces the pseudoscalar into the role of a pseudo-Goldstone boson, and hence to become massless. The mixing angle $\alpha$ is close to zero in all cases.

Together with the large $\tan \beta$ values, this ensures that the type II alignment limit condition of $|\beta-\alpha| \sim \frac{\pi}{2}$ (cf. Sect. 2.3) is always met, as is shown in Fig. 4. 


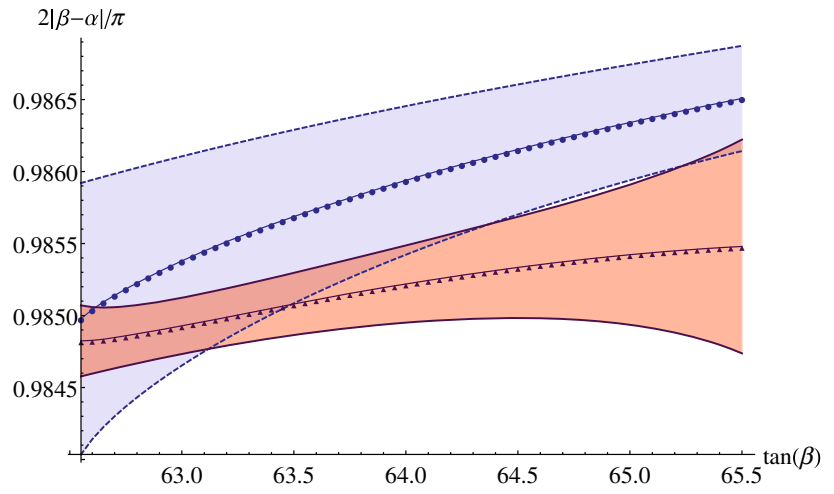

Fig. 4 Mixing angle difference $|\beta-\alpha|$ with difference between oneloop and two-loop fixed point results as uncertainty. The vacuum-stable and -unstable branches are marked as labelled in Fig. 1

To summarise, the mass spectrum produced by the solutions to the fixed point equations in the $\mathbb{Z}_{2}$-symmetric $2 \mathrm{HDM}$ exhibits a potential SM-Higgs candidate in the vacuumunstable branch, but is excluded by experimental observations because of the remaining boson spectrum. Both the light CP-even scalar and the charged Higgs boson are clearly too light to present a phenomenologically viable model. We fix this problem in the next section. Performing the same analysis in a type-Y 2 HDM leads to generally analogous results at slightly elevated $\tan \beta$-values.

\subsection{Vacuum stability of the fixed point solutions}

As alluded to in Sect. 2.2, further steps need to be taken to answer the question of vacuum stability beyond the first broad classification given by (2.9). While the following discussions are presented using the example of the fixed point solutions in the $\mathbb{Z}_{2}$-symmetric model, the results can easily be generalised and hold true for the softly-broken model as well.

In the case of the vacuum-stable branch of solutions, the $2 \mathrm{HDM}$ potential is bounded from below at all scales. However, it is not yet ensured that the minimum at $v=246 \mathrm{GeV}$ is the global minimum, or if there is another, deeper one. Since all couplings are known, checking condition (2.10) is only a matter of plugging in the values at the correct scale. We find that our stable branch solutions all satisfy Eq. (2.10) easily. While there usually exists another normal minimum, the SM-like minimum is always the deeper, global one.

In the case of the vacuum-unstable solutions, the situation turns out to be slightly more complex. With growing values of $\tan \beta, \lambda_{2}$ at the fixed point can become negative. Examining the RG evolution of $\lambda_{2}(\mu)$ to low scales shows that it only becomes negative at high energy scales. The instability scales for different values of $\tan \beta$ are shown in Fig. 5 (left plot). As can be inferred from that plot, the instability issue becomes worse with growing $\tan \beta$.
This behaviour is reminiscent of the SM quartic coupling. To decide if the models are really vacuum-unstable, the lifetime of these vacua has to be compared to the age of the universe. The tunnelling probability is calculated by using a semiclassical approximation [67], given as

$p \simeq\left(\frac{T_{U}}{R}\right)^{4} e^{-S_{0}}$

where $T_{U}$ is the age of the universe taken as $T_{U} \simeq 10^{10}$ years, $S_{0}$ is the Euclidean bounce action, and $R$ is a dimensional factor. We combine our two-loop RG-improved running couplings with a one-loop Coleman-Weinberg effective potential approximation. A very detailed account of such calculations performed for the SM case to a much higher degree of precision is given in $[67,68]$, a similar approach has been applied to the 2HDM in [69]. The effective potential can be approximated as

$V_{e f f}\left(\varphi_{1}, \varphi_{2}\right) \simeq \frac{\lambda_{2}^{e f f}}{8} \varphi_{2}^{4}$,

with $\lambda_{2}^{e f f}$ absorbing all one-loop terms $V_{e f f}^{1 l}$, using the assumption that $\varphi_{2}$ contributes dominantly. All squared mass terms in $V_{\text {eff }}$ can be expressed through the known running couplings, which are evaluated at the scale $\varphi_{2}=\mu$.

The result is shown in Fig. 5 (right) for the value of $\tan \beta=$ 66, which was the most unstable point in our solution space. The purple line marks the metastability bound, below which the tunnelling probability (4.4) becomes larger than one, and a vacuum catastrophe is expected to take place. The blue band shows the full evolution of $\lambda_{2}^{\text {eff }}$, evaluated at two-loop $\mathrm{RG}$ and one-loop effective potential level. Even in this "most unstable" case, it stays above the instability region.

We find that in our fixed point solutions, the boundedfrom-below conditions (2.9) are not broken severely enough to really make the model absolutely unstable. Instead, the situation seems to mirror the SM case: in our models, the solutions labelled "unstable" really fall in the metastability region. They do however cut quite close to the instability boundary.

It should be noted that especially compared to the SM, the 2HDM calculations presented above do not yet operate with the same degree of precision. Comparing one-loop and two-loop results in the both plots of Fig. 5 suggests that the theoretical uncertainties are too large to make concrete statements about vacuum stability. A full analysis combining the different approximations consistently to a higher order could be necessary and very interesting.

For this reason as much as for clarity's sake, we will continue to employ the terminology of "stable" and "unstable" in this work based on whether the conditions (2.9) are fulfilled when distinguishing between branches of fixed point 


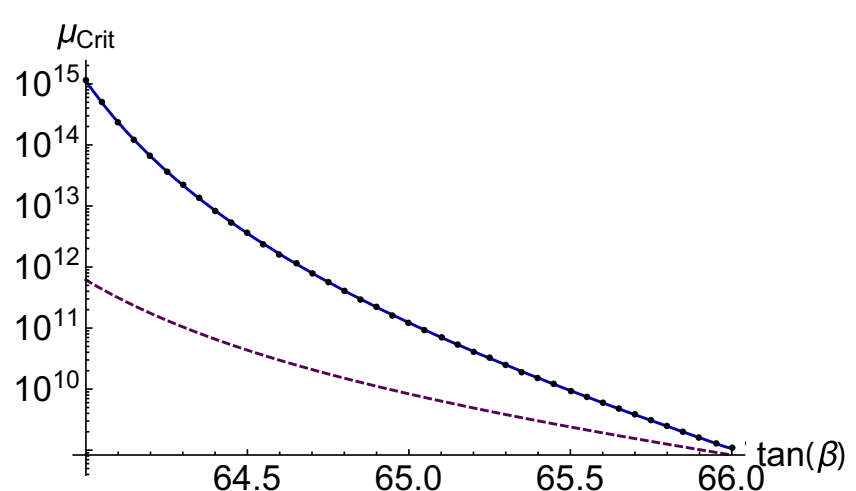

Fig. 5 Left: scale $\mu_{\text {crit }}$ at which the quartic coupling $\lambda_{2}(\mu)$ turns negative as a function of $\tan \beta$. The two-loop RG improved $\lambda_{2}^{2 l}(\mu)$ is shown as a blue line, the one-loop result as comparison is shown dashed in purple. Right: RG flow of the full quartic coupling $\lambda_{2}^{e f f}(\mu)$ (blue line)

solutions, regardless of their precise nature as discussed in this section.

\section{The $2 \mathrm{HDM}$ with softly broken $\mathbb{Z}_{2}$}

In Sect. 4 we have found fixed points, but the resulting models are all experimentally excluded due to small mass values. To allow for larger, phenomenologically viable masses for $m_{h}$, $m_{H^{+}}$, and $m_{A}$, it is necessary to go beyond the $\mathbb{Z}_{2}$-symmetric model. The least invasive way to generate heavier masses is to include the so-called softly-broken $\mathbb{Z}_{2}$-symmetric $2 \mathrm{HDMs}$. The assumption of a $\mathbb{Z}_{2}$-symmetry under the transformation $\Phi_{1} \rightarrow-\Phi_{1}$ is not completely dropped, but a mass term $M_{12}^{2}\left(\Phi_{1}^{\dagger} \Phi_{2}+\Phi_{2}^{\dagger} \Phi_{1}\right)$ mixing between the two Higgs field is allowed.

Like the other mass parameters, $M_{12}$ does not appear in any quartic, gauge, or Yukawa beta function. It can instead be treated as a free parameter. For this reason, $M_{12}$ does not influence the fixed point search itself. Phenomenologically, on the other hand, the mixing parameter can have a big impact, especially in the case of $\lambda_{5}=0$ observed in our models. The additional global U(1) symmetry is now broken by non-vanishing $M_{12}$-terms, which allows the pseudoscalar Higgs boson to acquire mass. Additionally, three of the other four bosons grow approximately linear with $M_{12}$, which allows them to evade experimental constraints. The influence of $M_{12}$ on the different boson masses for the vacuum-unstable branch is illustrated in Fig. 6. The behaviour is analogous in the vacuum-stable branch.

As can be seen in the plot, the CP-even neutral scalar eigenvalues (blue/violet) depend on $M_{12}$ in different ways [cf. Eq. (2.4)]. The SM-like eigenstate (originally $m_{H}$ ) only shows a minor dependence, and hardly changes even for very large values of $M_{12}$. For the originally smaller eigenstate however, $M_{12}$ can easily become the dominating contributor.

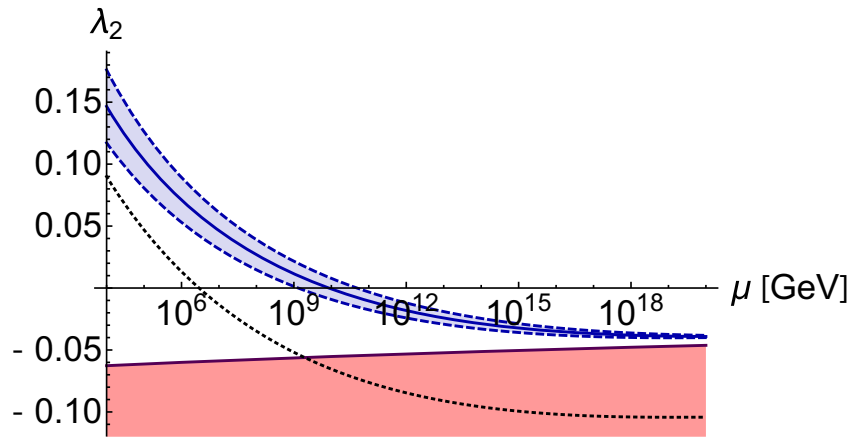

for $\tan \beta=66$. The purple boundary marks the metastability/instability boundary, with the unstable side shaded. The blue dashed region is given by the size of the contribution of the one-loop effective potential. The black dotted line shows the naked one-loop RG running of $\lambda_{2}^{1 l}$

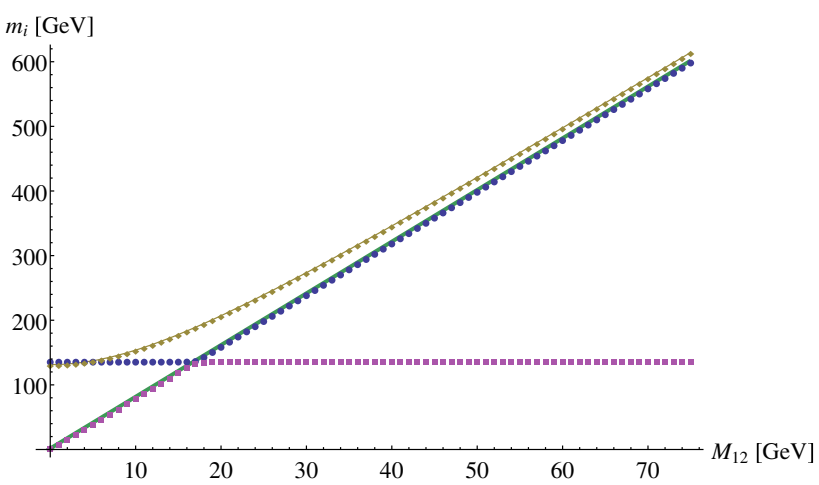

Fig. 6 Higgs boson masses for a softly broken $\mathbb{Z}_{2}$-symmetry for $\tan \beta=64$ against $M_{12}$ in $\mathrm{GeV}$ for the vacuum-unstable branch of solutions. The masses shown correspond to the CP-even scalars $m_{h}$, $m_{H}$ (violet squares, blue circles), the charged Higgs $m_{H^{+}}$(yellow diamonds) and the pseudoscalar $m_{A}$ (green line)

While the original mass of this state was mainly generated by a small VEV $v_{2}$, it soon starts to grow almost linearly with $M_{12}$, surpassing the mass of the former heavier eigenstate in a level crossing at values of roughly $M_{12} \approx 20 \mathrm{GeV}$.

Both the charged Higgs (yellow) and the pseudoscalar Higgs (green) also grow together with $M_{12}$, and adopt an asymptotically linear behaviour as $M_{12}$ becomes large. For the pseudoscalar, the linear dependence is actually exact as long as $\lambda_{5}=0$, as it is the case with our fixed point solutions. The mass eigenvalue in (2.7) then simplifies to:

$m_{A}=\frac{M_{12}}{\sqrt{\sin \beta \cos \beta}} \sim M_{12} \sqrt{\tan \beta}$.

For this reason the pseudoscalar mass $m_{A}$ in Fig. 6 is shown as a straight line.

Compared to $M_{12}, \tan \beta$ has only a minor impact on the heavy boson masses. Figure 7 shows the SM-like $M_{12-}$ independent eigenstate, henceforth referred to as $m_{h}$, with 
the vacuum-stable case on the left and the vacuum-unstable case on the right. The distortion around $M_{12} \sim 18 \mathrm{GeV}$ arises from the point where the $\mathrm{CP}$-even eigenvalues become degenerate. Figure 8 shows the corresponding plots for the $M_{12}$-dependent CP-even eigenstate $m_{H}$ on the left for $m_{H^{+}}$ on the right. Displayed in the figure is the vacuum stable case; the differences to the vacuum-unstable solution branch is absolutely minimal. The SM-like Higgs in Fig. 7 is the only eigenstate for which there remains a significant difference in stable and unstable branch. The pseudoscalar mass is not shown. The behaviour of $m_{A}$ is nearly indistinguishable from $m_{H}$ in these regions, as demonstrated already in Fig. 6. For the heavy bosons, contour lines are drawn in $25 \mathrm{GeV}$ Intervals.

A finite $M_{12}$ has a number of implications on the validity of the theory. Most importantly, it has the anticipated effect of allowing the model to produce phenomenologically viable mass spectra by opening up a way to drive $m_{h}, m_{A}$ and $m_{H^{+}}$ to higher values. The experimental bounds on the physical Higgs bosons can be translated to a lower bound on $M_{12}$. From the bound of $m_{H^{+}}>580 \mathrm{GeV}$ [55] in type-Y models it follows that:

$M_{12} \gtrsim 70 \mathrm{GeV}$

with the exact value depending on $\tan \beta$. Re-translated, this condition implies in terms of other boson masses:

$m_{A}, m_{H} \geq 550 \mathrm{GeV}$.

Because of the mixing angle $\alpha$ being close to zero, the SMlike Higgs mass stays almost unchanged. This means that in terms of vacuum stability, the situation also remains consistent with the $\mathbb{Z}_{2}$-symmetric case: While there are vacuumstable solutions to the fixed point equations, only the vacuumunstable ones include masses around $125 \mathrm{GeV}$. The SM-like Higgs is thus in a unique position among the 2HDM bosons, in that its mass cannot be heavily adjusted in this model.

Whereas the SM-like CP-even scalar eigenstate is independent of $M_{12}$, the opposite is true for all other bosons: Even at its minimum, $M_{12} \sim 70 \mathrm{GeV}$ is already large enough to make it the controlling factor in generating the masses of the three bosons $H, H^{+}$and $A$. For larger values of $M_{12}$, the degeneracy in masses becomes even stronger. Therefore, most of the parameter space of viable asymptotically safe 2HDMs falls into the decoupling limit [57], with one SMlike and three heavy bosons with $m_{H} \approx m_{H^{+}} \approx m_{A} \propto M_{12}$.

The high $\tan \beta$-values necessary to find fixed points mean that in type II models specifically, bounds from $B_{s} \rightarrow \mu \mu$ decays are much more restrictive $[56,70]$. They demand heavy boson masses upwards of

$m_{H} \approx m_{H^{+}} \approx m_{A}>3 \mathrm{TeV}$.

\subsection{Stability analysis}

In order to understand the characteristics of a given fixed point, we study the linearised RG flow around the fixed point, described by the stability matrix given by:

$M_{i j}=\left.\frac{\partial \beta_{i}}{\partial g_{j}}\right|_{F P}$.

In this case, $g_{j}$ includes gauge, Yukawa and quartic couplings. The number of negative eigenvalues of $M_{i j}$ corresponds to the dimension of the critical surface from which trajectories run into the fixed point. However, it has less significance here: While it is important to confirm that the fixed points are indeed UV-attractive (which they are), both the exact fixed point scale and the low scale initial conditions give additional constraints that intersect non-trivially with the critical surface. The solution to Eq. (3.1) is always a single trajectory in parameter space. On the other hand, by construction our method of finding fixed points ensures that the solutions found connect to the critical surface.

It is therefore necessary to examine which of the initial conditions used is subject to uncertainties, and how these translate to changes in fixed point solutions and thus in Higgs boson mass spectra.

\subsection{Uncertainty estimates}

There are several factors that influence the fixed point analysis. Below, we look at changes in scale where the fixed point condition is applied, followed by a discussion about low scale top and bottom quark mass uncertainties. Unless stated differently, values for the SM-like CP-even scalar eigenstate (here $h$ ) will be evaluated using $M_{12, \text { Min }}=380 \mathrm{GeV}$. The pseudoscalar $A$ and $M_{12}$-dependent $\mathrm{CP}$-even scalar eigenstate $H$ are entirely or almost entirely generated by the free parameter $M_{12}$ and therefore have negligible uncertainties.

In general, the models are studied with the condition that the quartic coupling beta functions become zero at $m_{P l}=1.2 \cdot 10^{19} \mathrm{GeV}$. The mass spectrum shows a minor dependence on where exactly the fixed points are assumed to occur. The masses $m_{h}$ are shown in Fig. 9 for different fixed point scales. We see that lower fixed point scales correspond to a larger difference between vacuum-stable (upper) and vacuum-unstable (lower) branches, and notably bring down the lower branch mass values. Also, the $\tan \beta$-range in which fixed points can be found changes with the fixed point scale: when the fixed points scale is chosen at higher values than $m_{P l}$, the divergence of large Yukawa couplings, especially $y_{b}$ becomes an even more pronounced problem.

The mass spectra depend on the initial values chosen for the gauge and Yukawa couplings. The dependency on the top quark mass turns out to be especially strong. In Fig. 10 the 

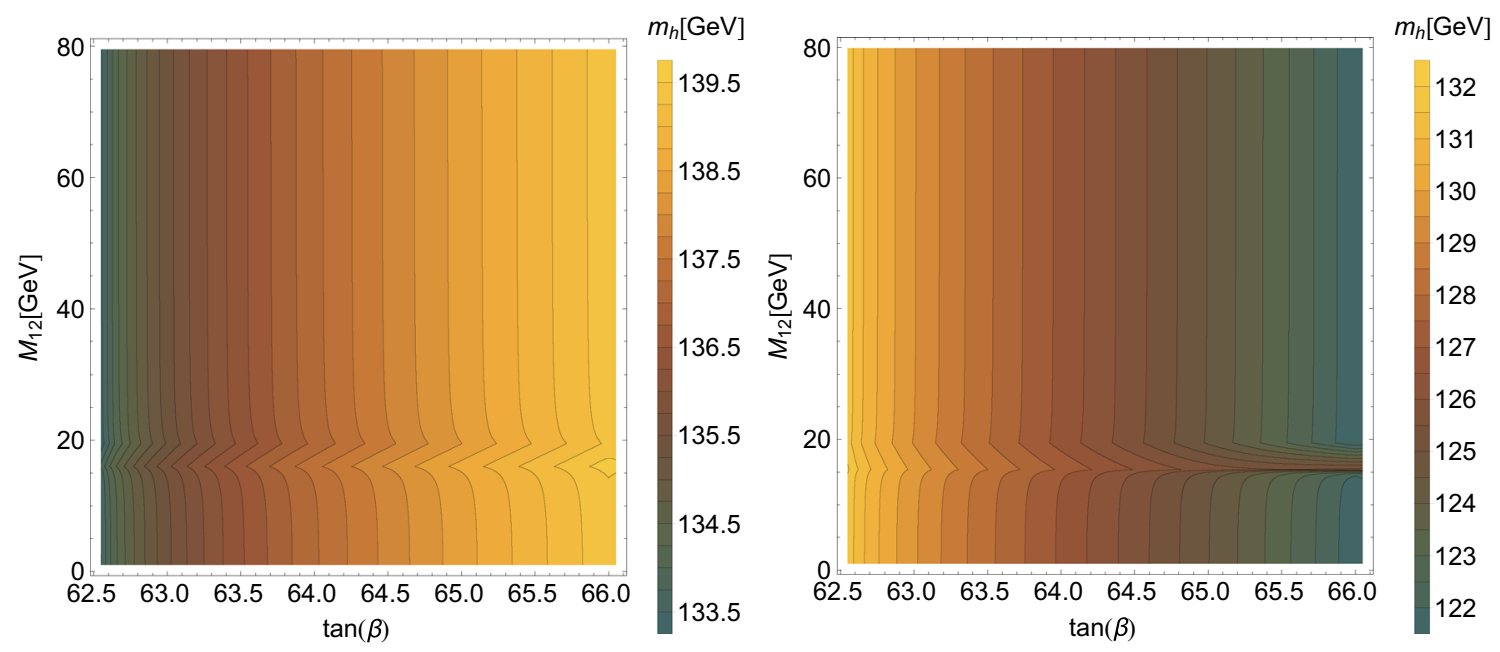

Fig. 7 Masses of $M_{12}$-independent CP-even neutral scalar $m_{h}$ as a function of $\tan \beta$ and the soft breaking parameter $M_{12}$. The vacuum-stable branch is shown on the left, the vacuum-unstable branch on the right
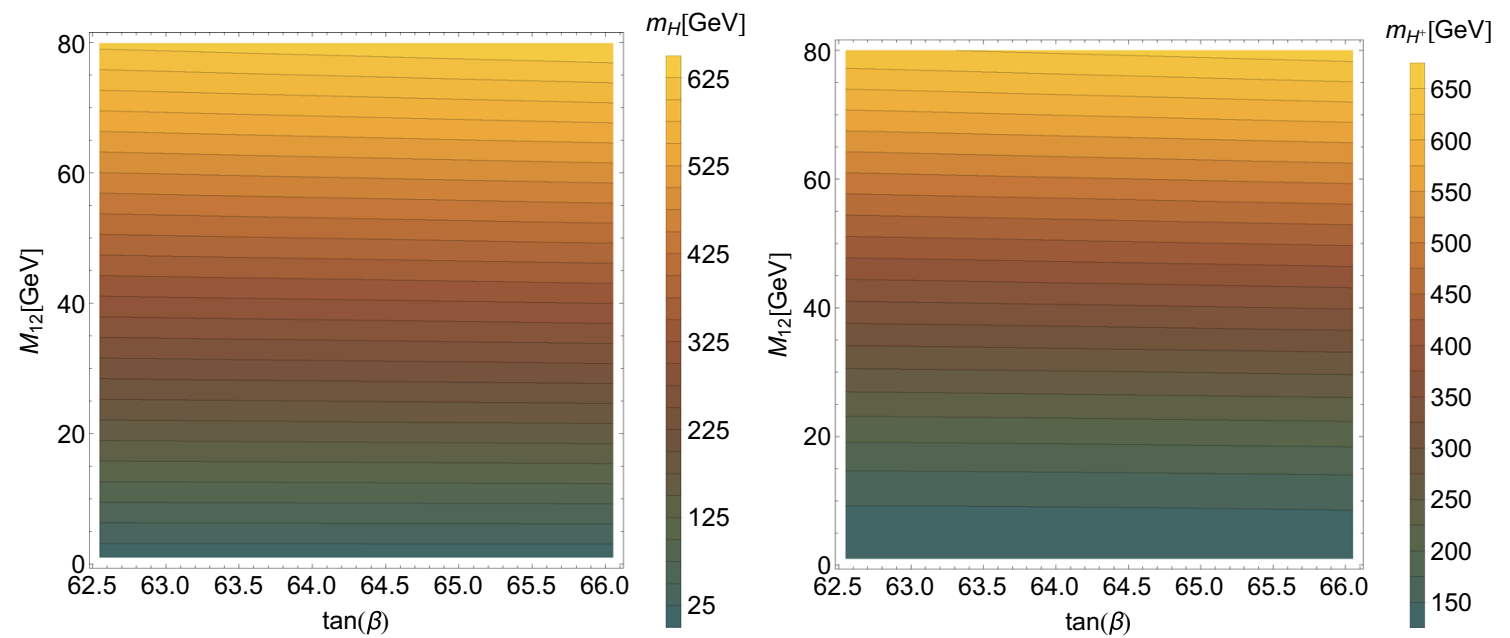

Fig. 8 Masses of $M_{12}$-dependent CP-even neutral scalar $m_{H}$ (left) and charged Higgs boson $m_{H^{+}}$(right) as a function of tan $\beta$ and the soft breaking parameter $M_{12}$

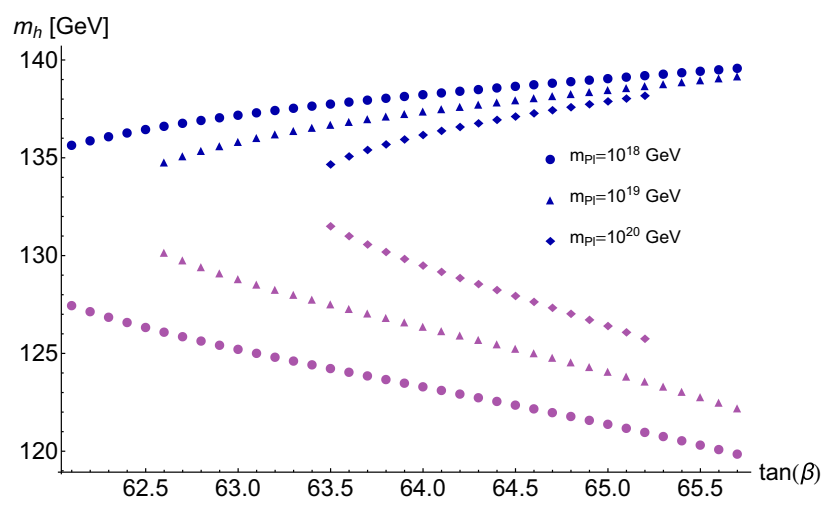

Fig. 9 Masses of SM-like CP-even scalar Higgs boson, for $M_{12}=380$ $\mathrm{GeV}$ and fixed point condition set at different scales of $10^{18}$ (circles), $10^{19}$ (triangles), and $10^{20}$ (diamonds) GeV. The upper (blue) branches correspond to the vacuum-stable solutions, the lower (purple) branches to the vacuum-unstable ones
SM-like Higgs boson masses are shown for the $1 \sigma$ deviation bands of the $\overline{M S}$ top and bottom quark masses of $m_{t}\left(m_{t}\right)=$ $\left(160_{-4.3}^{+4.8}\right) \mathrm{GeV}$ and $m_{b}\left(m_{b}\right)=(4.18 \pm 0.03) \mathrm{GeV}$ [64]. For the charged Higgs mass, the uncertainty generally grows with $\tan \beta$, but also depends on the soft breaking parameter, as the quartic coupling contributions to the boson mass weaken with ${\sqrt{M_{12}}}^{-1}$. As such, the influence of quark initial values on the uncertainties becomes smaller with growing heavy boson masses. The absolute sizes of uncertainty bands $\Delta m_{H^{+}}$are shown against the central mass value $m_{H^{+}}$in Fig. 11. The vertical width of the bands in this plot indicates how $\Delta m_{H}^{+}$ depends on $\tan (\beta)$. It has to be noted that the $\tan \beta$-interval shown in the first (left) plots of Figs. 10 and 11 is smaller than the intervals shown in the corresponding right hand plot, or in Figs. 1, 2, 3, 4: the reason is that different Yukawa initial values not only change the mass spectrum, but also the region 
in parameter space for which a fixed point exists: On one hand, smaller quark masses mean that the Yukawa couplings require larger $\tan \beta$ to fulfil the fixed point condition. On the other hand, larger quark masses move the Landau pole of the Yukawa couplings to lower scales. The first effect is very noticeable for lower values of $m_{t}$. This effect is also a reason why the variance in uncertainties at fixed values of $M_{12}$ is much smaller in the left plot of Fig. 11 compared to the right one: As fixed points for all top quark initial values can only be found over a very small interval of $\tan (\beta)$, the influence on of $\tan (\beta)$ on $m_{H}^{+}$at a fixed value of $M_{12}$ stays limited.

Figure 12 shows a typical set of running quartic couplings each for the vacuum-stable and unstable branch and how different top quark mass initial values influence $\lambda_{i}$. For most couplings, the $m_{t}$-induced relative uncertainty becomes smaller as the scale decreases, even if the coupling itself becomes larger. This is most notable for $\lambda_{1}$ (blue) and $\lambda_{2}$ (violet). The quartic coupling $\lambda_{5}$ is not shown in these graphs, as $\lambda_{5}=0$ is an exact solution of the fixed point equations regardless of initial conditions. A last important detail to take note of is the range of $\lambda_{2}$ in the right graph: depending on the exact initial conditions, $\lambda_{2}$ may become negative (and thereby break the vacuum stability conditions of Eq. (2.9)) as early as $10^{8} \mathrm{GeV}$, or not at all. To further analyse the influence of the exact starting parameters on the Higgs mass, all SM-like Higgs mass values generated by a fixed point solution can be shown against the corresponding top quark Yukawa initial value. Figure 13 shows the Higgs mass $m_{h}$ for the $1 \sigma$ regions of $m_{b}$ and $m_{t}$ for two different values of $\tan \beta$. The vertical spread in points is generated by shifting the bottom quark initial value. Once again, all vacuum-stable points are coloured blue, the vacuum-unstable ones violet. The right hand graph in particular illustrates well the two branches of fixed point solutions: The branch corresponding to higher Higgs boson masses is fully vacuum-stable, whereas the lower mass branch can only be vacuum-stable for top quark mass values on the lower end of its $1 \sigma$ band. Comparing both graphs also shows how $\tan \beta$ needs to be of certain size to facilitate the existence of fixed points. For the central mass values of $m_{b}$ and $m_{t}$, there are no fixed points at $\tan \beta=60$. However, as shown in the left graph, fixed points can be found there if either or both initial values are slightly larger.

\section{Summary}

Proposing simultaneously vanishing quartic coupling beta functions at the Planck scale severely constrains the 2HDM parameter space, but is possible in a way similar to the SM. As such, the 2HDM likewise supports the idea of being extended to high scales through means of asymptotic safety.
The parameter $\tan \beta$ needs to be large, as both the up-type and the down-type Yukawa couplings have to be large in order to keep the positive contributions in quartic couplings beta functions in check. For the same reason, only type II and type Y models are viable, while type I and type X models are not. In the type II/type $\mathrm{Y}$ models studied, there always exists a $\tan \beta$-interval in which fixed points can be found, see Eq. (4.3). Fixed points can be found in $\mathbb{Z}_{2}$-symmetric models, but the resulting mass spectra are not phenomenologically viable. The most minimal model that also agrees with all experimental bounds is the softly-broken $\mathbb{Z}_{2}$-symmetric 2HDM, cf. Figs. 6, 7 and 8.

The allowed parameter region defined by the fixed point assumption meets the characteristics of the decoupled alignment limit, with three heavy Higgs bosons $m_{H} \approx$ $m_{A} \approx m_{H^{+}} \propto M_{12}$, and $|\beta-\alpha| \approx \frac{\pi}{2}$. To be consistent with experimental constraints, a lower bound is given on $M_{12}>70 \mathrm{GeV}(380 \mathrm{GeV}$ ) for type Y (type II) models, corresponding to the charged Higgs limits for type Y. This implies lower limits on $m_{A}$ and $m_{H}$, see Eqs. (5.3) and (5.4).

Similar to the SM, both the existence of fixed points and the vacuum stability depend strongly on the low scale initial values, most notably the exact top quark mass. Even more similar to the SM is the fact that while the boundedfrom-below conditions (2.9) are sometimes violated in fixed point solutions, the violation only happens at high scales, and is mostly weak enough for the potential to remain in a metastable region, cf. Fig. 5. As illustrated in Fig. 13, the central values of Higgs and top quark mass indicate an unstable/metastable vacuum, but are rather close to the criticality border. Fixed point solutions with absolute stability can be possible by having $\overline{\mathrm{MS}}$ top quark mass values lower than 160 $\mathrm{GeV}$ [64] or the fixed point scale set below $m_{P l}=1.2 \cdot 10^{19}$ $\mathrm{GeV}$. A more precise determination of $m_{t}$ and $m_{h}$ by future experiments will allow a more definite statement.

In the end, the fact that Asymptotic Safety in the 2HDM is supported exactly in the parameter space region in which the 2HDM behaves most SM-like is intriguing. However, the extremely high values of $\tan \beta$ necessary to make fixed points work only just allow the model to exist in accordance with current experimental bounds. It would be interesting to see how the 2HDM behaves when combined with the types of SM-extensions that can support more complete UV-interactive fixed points in the gauge-Yukawa sector. An extended (possibly dark) fermion sector in particular could allow for easier model building, if it can lift some of the burden put on the SM Yukawa couplings by the fixed point conditions, and by extension on $\tan \beta$.

As it stands, the 2HDM does not solve the stability problem of the SM. Instead, the situation there too is a mirror to the SM. The branches of fixed point solutions with promising SM-like Higgs mass candidates seem to correspond to metastable vacuum potentials. A thorough NNLO analysis 

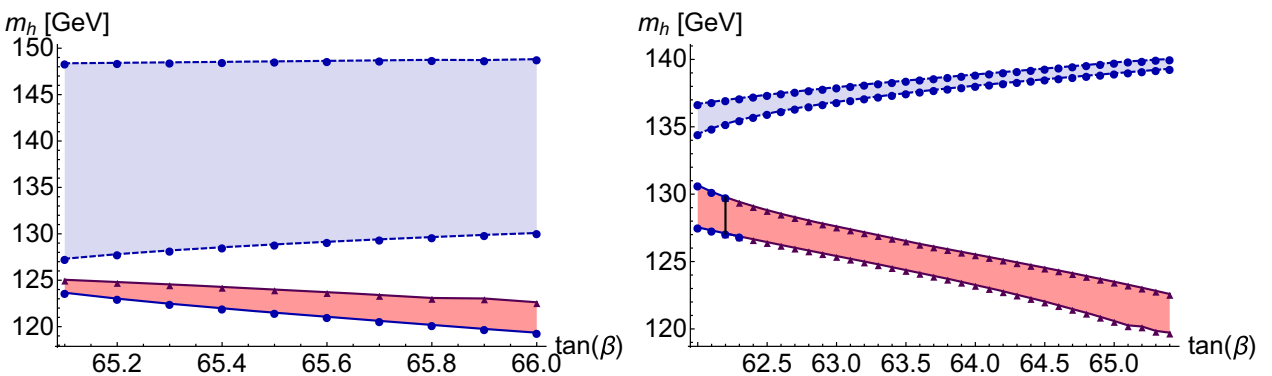

Fig. 10 Masses of SM-like CP-even Higgs boson evaluated at $M_{12}=380 \mathrm{GeV}$ with $1 \sigma$ uncertainty regions from top quark (left) and bottom quark (right) mass initial values of $\left(160_{-4.3}^{+4.8}\right) \mathrm{GeV}$ and $(4.18 \pm 0.03) \mathrm{GeV}$ respectively. Stable solutions are marked in blue with dashed outline, unstable solutions in violet

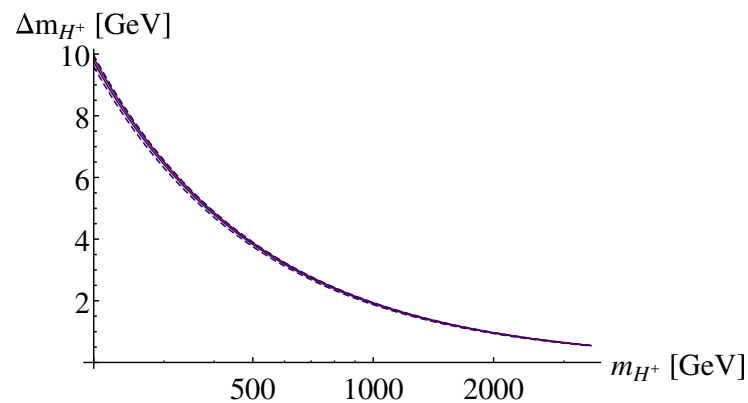

Fig. 11 Dependence of the sizes of the uncertainty bands $\Delta m_{H^{+}}$on the charged Higgs mass when driven by $M_{12}$. The left (right) plot shows the size of $\Delta m_{H^{+}}$generated by the $1 \sigma$ top (bottom) quark uncertainty.

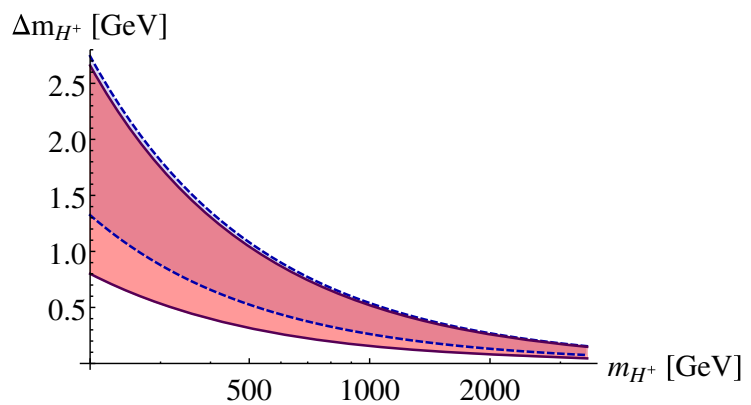

Stable solutions are marked in blue with dashed lines, unstable solutions in violet. The width of the bands is the variance in uncertainty generated by working at different values of $\tan (\beta)$
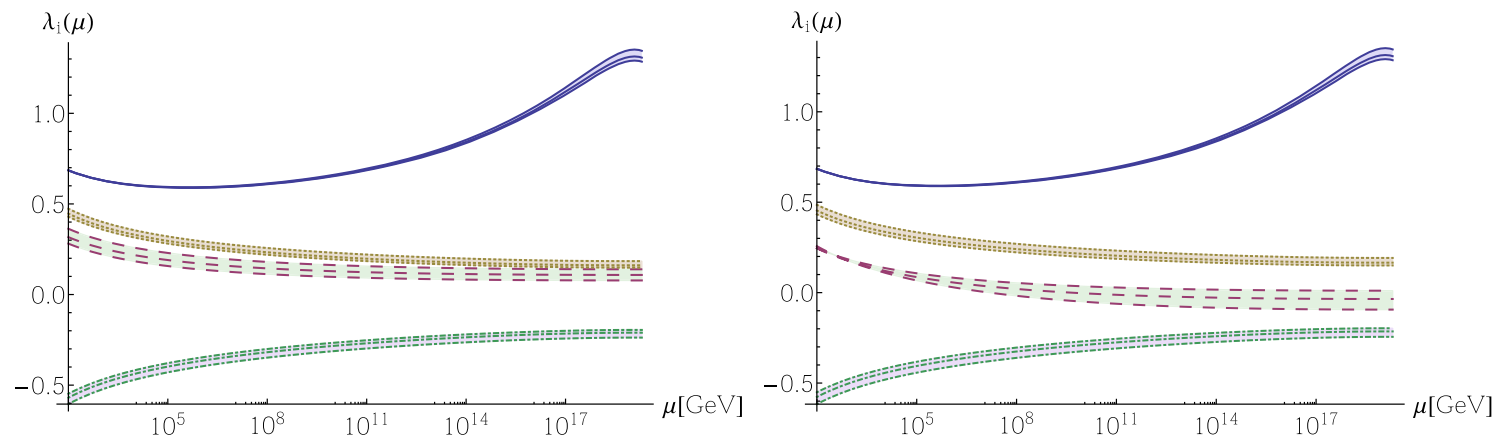

Fig. 12 Running quartic couplings with $1 \sigma$ uncertainty intervals from low scale top quark mass initial value for the vacuum-stable (left) and vacuum-unstable (right) fixed point branch. The colours correspond to: $\lambda_{1}$ (blue), $\lambda_{2}$ (violet, dashed), $\lambda_{3}$ (yellow, dotted) and $\lambda_{4}$ (green, dot-dashed)
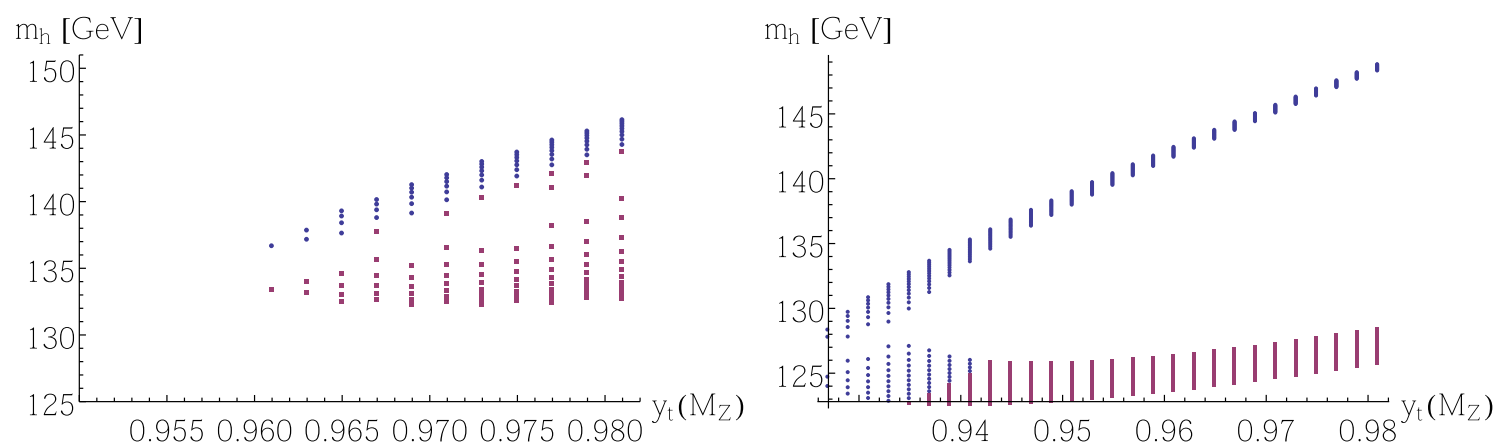

Fig. 13 Mass region of the SM-like CP-even scalar Higgs boson from $1 \sigma$ quark mass uncertainties against the top Yukawa initial value $y_{t}\left(M_{Z}\right)$ for $\tan \beta=60$ (left) and $\tan \beta=64$ (right). Stable solutions are marked in blue, unstable solutions in violet 
of the metastability border may be helpful to achieve clarity in this matter.

Note added: During the final phase of this project, an analysis of 2HDM fixed points using slightly different methodology appeared [71]. While our approach differs in details, we agree with the general conclusion that fixed points in type II 2HDMs are possible.

Acknowledgements We are happy to thank Gudrun Hiller for fruitful discussions, and Otto Eberhardt for helpful comments.

Data Availability Statement This manuscript has no associated data or the data will not be deposited. [Authors' comment: There is no relevant data that could be deposited for this manuscript. The work presented is of theoretical nature, and the calculations can be performed without additional input.]

Open Access This article is distributed under the terms of the Creative Commons Attribution 4.0 International License (http://creativecomm ons.org/licenses/by/4.0/), which permits unrestricted use, distribution, and reproduction in any medium, provided you give appropriate credit to the original author(s) and the source, provide a link to the Creative Commons license, and indicate if changes were made.

Funded by SCOAP ${ }^{3}$.

\section{Appendix: $\beta$ functions}

In this section, the $\beta$ functions $\beta_{g_{i}}=\frac{d g_{i}(\mu)}{d \log (\mu)}=\mu \frac{d g_{i}(\mu)}{d \mu}$ are listed on 2-loop level for the most general model used (i.e. the softly-broken $\mathbb{Z}_{2}$-symmetric 2 HDM type II, which means that $\lambda_{6}$ and $\lambda_{7}$ do not appear). They were calculated with the Mathematica package SARAH $[61,62]$. The general procedure of how to derive 2-loop RGEs for general field theories has been outlined in [72-74].

In the case of Yukawa couplings, first and second generation contributions have been neglected. The Yukawa matrices have therefore been restricted to their respective $(3,3)$ entries.

The $\beta$ functions for the gauge couplings are given by:

$$
\begin{aligned}
16 \pi^{2} \beta_{g_{1}}= & 7 g_{1}^{3}+\frac{1}{288 \pi^{2}}\left(g _ { 1 } ^ { 3 } \left(208 g_{1}^{2}+3\left(36 g_{2}^{2}\right.\right.\right. \\
& \left.\left.\left.+88 g_{3}^{2}-5 \lambda_{b}^{2}-15 \lambda_{\tau}^{2}-17 \lambda_{t}^{2}\right)\right)\right) \\
16 \pi^{2} \beta_{g_{2}}= & -3 g_{2}^{3}+\frac{1}{32 \pi^{2}}\left(-g_{2}^{3}\left(-4 g_{1}^{2}-16 g_{2}^{2}\right.\right. \\
& \left.\left.-24 g_{3}^{2}+3 \lambda_{b}^{2}+\lambda_{\tau}^{2}+3 \lambda_{t}^{2}\right)\right) \\
16 \pi^{2} \beta_{g_{3}}= & -7 g_{3}^{3}+\frac{1}{96 \pi^{2}}\left(-g_{3}^{3}\left(3 \left(4 \left(13 g_{3}^{2}+\lambda_{b}^{2}\right.\right.\right.\right. \\
& \left.\left.\left.\left.+\lambda_{t}^{2}\right)-9 g_{2}^{2}\right)-11 g_{1}^{2}\right)\right)
\end{aligned}
$$

The $\beta$ functions for the quartic Higgs couplings $\lambda_{i}$ for the softly-broken type II $2 \mathrm{HDM}$ are given by:

$$
16 \pi^{2} \beta_{\lambda_{1}}=\frac{1}{4}\left(6 g_{1}{ }^{2}\left(g_{2}{ }^{2}-2 \lambda_{1}\right)+3 g_{1}{ }^{4}-36 g_{2}{ }^{2} \lambda_{1}+9 g_{2}{ }^{4}\right.
$$

$$
\begin{aligned}
& +8\left(2 \lambda_{1}\left(3 \lambda_{b}{ }^{2}+\lambda_{\tau}^{2}\right)+6 \lambda_{1}^{2}+2 \lambda_{3} \lambda_{4}\right. \\
& \left.\left.+2 \lambda_{3}{ }^{2}+\lambda_{4}{ }^{2}+\lambda_{5}{ }^{2}-6 \lambda_{b}{ }^{4}-2 \lambda_{\tau}{ }^{4}\right)\right) \\
& +\frac{1}{384 \pi^{2}}\left[g _ { 1 } ^ { 2 } \left(6 g _ { 2 } ^ { 2 } \left(39 \lambda_{1}+20 \lambda_{4}\right.\right.\right. \\
& \left.+36 \lambda_{b}^{2}+44 \lambda_{\tau}^{2}\right)-303 g_{2}^{4} \\
& +4\left(25 \lambda_{1}\left(\lambda_{b}{ }^{2}+3 \lambda_{\tau}^{2}\right)+108 \lambda_{1}^{2}\right. \\
& +4\left(6\left(2 \lambda_{3} \lambda_{4}+2 \lambda_{3}^{2}+\lambda_{4}^{2}\right)\right. \\
& \left.\left.\left.-3 \lambda_{5}{ }^{2}+4 \lambda_{b}{ }^{4}-12 \lambda_{\tau}{ }^{4}\right)\right)\right)+g_{1}{ }^{4}\left(-573 g_{2}{ }^{2}\right. \\
& \left.+651 \lambda_{1}+60\left(2 \lambda_{3}+\lambda_{4}+\lambda_{b}{ }^{2}-5 \lambda_{\tau}^{2}\right)\right) \\
& -393 g_{1}{ }^{6}+3\left(-3 g_{2}{ }^{4}\left(17 \lambda_{1}\right.\right. \\
& \left.+4\left(-10 \lambda_{3}-5 \lambda_{4}+3 \lambda_{b}^{2}+\lambda_{\tau}^{2}\right)\right) \\
& +12 g_{2}{ }^{2}\left(5 \lambda_{1}\left(3 \lambda_{b}{ }^{2}+\lambda_{\tau}{ }^{2}\right)+36 \lambda_{1}{ }^{2}\right. \\
& \left.+4\left(2 \lambda_{3}+\lambda_{4}\right)^{2}\right)+291 g_{2}{ }^{6} \\
& -8\left(\lambda _ { 1 } \left(-80 g_{3}{ }^{2} \lambda_{b}{ }^{2}+20 \lambda_{3} \lambda_{4}+20 \lambda_{3}{ }^{2}\right.\right. \\
& \left.+12 \lambda_{4}^{2}+14 \lambda_{5}^{2}+3 \lambda_{b}^{4}+\lambda_{\tau}^{4}\right) \\
& +4\left(16 g_{3}{ }^{2} \lambda_{b}{ }^{4}+\lambda_{5}{ }^{2}\left(10 \lambda_{3}+11 \lambda_{4}\right)\right. \\
& +8 \lambda_{3} \lambda_{4}{ }^{2}+6 \lambda_{3}{ }^{2} \lambda_{4} \\
& \left.+4 \lambda_{3}{ }^{3}+3 \lambda_{4}{ }^{3}-5\left(3 \lambda_{b}{ }^{6}+\lambda_{\tau}{ }^{6}\right)\right) \\
& \left.+24 \lambda_{1}^{2}\left(3 \lambda_{b}{ }^{2}+\lambda_{\tau}^{2}\right)+78 \lambda_{1}^{3}\right) \\
& -24 \lambda_{t}^{2}\left(3 \lambda_{1} \lambda_{b}^{2}+8 \lambda_{3} \lambda_{4}+8 \lambda_{3}^{2}\right. \\
& \left.\left.\left.+4\left(\lambda_{4}^{2}+\lambda_{5}^{2}\right)-4 \lambda_{b}^{4}\right)\right)\right] \text {. }
\end{aligned}
$$

$$
\begin{aligned}
16 \pi^{2} \beta_{\lambda_{2}}= & \frac{1}{4}\left(6 g_{1}{ }^{2}\left(g_{2}{ }^{2}-2 \lambda_{2}\right)+3 g_{1}{ }^{4}-36 g_{2}{ }^{2} \lambda_{2}+9 g_{2}{ }^{4}\right. \\
& +8\left(6 \lambda_{2} \lambda_{t}{ }^{2}+6 \lambda_{2}{ }^{2}+2 \lambda_{3} \lambda_{4}+2 \lambda_{3}{ }^{2}\right. \\
& \left.\left.+\lambda_{4}{ }^{2}+\lambda_{5}{ }^{2}-6 \lambda_{t}{ }^{4}\right)\right) \\
& +\frac{1}{384 \pi^{2}}\left[g _ { 1 } { } ^ { 2 } \left(6 g _ { 2 } { } ^ { 2 } \left(39 \lambda_{2}+20 \lambda_{4}\right.\right.\right. \\
& \left.+84 \lambda_{t}{ }^{2}\right)-303 g_{2}{ }^{4} \\
& +48\left(9 \lambda_{2}{ }^{2}+4 \lambda_{3} \lambda_{4}+4 \lambda_{3}{ }^{2}+2 \lambda_{4}{ }^{2}\right. \\
& \left.\left.-\lambda_{5}{ }^{2}\right)+340 \lambda_{2} \lambda_{t}{ }^{2}-128 \lambda_{t}{ }^{4}\right) \\
& +3 g_{1}{ }^{4}\left(-191 g_{2}{ }^{2}+217 \lambda_{2}+40 \lambda_{3}\right. \\
& \left.+20 \lambda_{4}-76 \lambda_{t}{ }^{2}\right)-393 g_{1}{ }^{6} \\
& +3\left(g_{2}{ }^{4}\left(-51 \lambda_{2}+60\left(2 \lambda_{3}+\lambda_{4}\right)-36 \lambda_{t}{ }^{2}\right)\right.
\end{aligned}
$$




$$
\begin{aligned}
& +12 g_{2}^{2}\left(4\left(9 \lambda_{2}^{2}+\left(2 \lambda_{3}+\lambda_{4}\right)^{2}\right)\right. \\
& \left.+15 \lambda_{2} \lambda_{t}^{2}\right)+291 g_{2}{ }^{6} \\
& +8\left(\lambda_{t}{ }^{4}\left(-64 g_{3}^{2}-3 \lambda_{2}+12 \lambda_{b}^{2}\right)\right. \\
& +\lambda_{2} \lambda_{t}^{2}\left(80 g_{3}^{2}-9\left(8 \lambda_{2}+\lambda_{b}^{2}\right)\right) \\
& -2 \lambda_{5}{ }^{2}\left(7 \lambda_{2}+20 \lambda_{3}+22 \lambda_{4}\right)-2\left(2 \lambda _ { 4 } ^ { 2 } \left(3 \lambda_{2}\right.\right. \\
& \left.+8 \lambda_{3}\right)+2 \lambda_{3} \lambda_{4}\left(5 \lambda_{2}+6 \lambda_{3}\right) \\
& \left.+10 \lambda_{2} \lambda_{3}{ }^{2}+39 \lambda_{2}{ }^{3}+8 \lambda_{3}^{3}+6 \lambda_{4}^{3}\right) \\
& -12 \lambda_{b}{ }^{2}\left(2 \lambda_{3} \lambda_{4}+2 \lambda_{3}{ }^{2}+\lambda_{4}{ }^{2}+\lambda_{5}^{2}\right) \\
& -4 \lambda_{\tau}^{2}\left(2 \lambda_{3} \lambda_{4}+2 \lambda_{3}{ }^{2}+\lambda_{4}{ }^{2}\right. \\
& \left.\left.\left.\left.+\lambda_{5}{ }^{2}\right)+60 \lambda_{t}{ }^{6}\right)\right)\right] .
\end{aligned}
$$

$$
\begin{aligned}
& 16 \pi^{2} \beta_{\lambda_{3}}=\frac{1}{4}\left(-6 g_{1}{ }^{2}\left(g_{2}{ }^{2}+2 \lambda_{3}\right)+3 g_{1}{ }^{4}\right. \\
& -36 g_{2}^{2} \lambda_{3}+9 g_{2}{ }^{4} \\
& +8\left(\lambda_{1}\left(3 \lambda_{3}+\lambda_{4}\right)+\lambda_{2}\left(3 \lambda_{3}+\lambda_{4}\right)\right. \\
& +\lambda_{3}\left(2 \lambda_{3}+3 \lambda_{b}^{2}+\lambda_{\tau}^{2}\right) \\
& \left.\left.+3 \lambda_{t}{ }^{2}\left(\lambda_{3}-2 \lambda_{b}{ }^{2}\right)+\lambda_{4}^{2}+\lambda_{5}^{2}\right)\right) \\
& +\frac{1}{384 \pi^{2}}\left[g _ { 1 } { } ^ { 2 } \left(6 g _ { 2 } { } ^ { 2 } \left(39 \lambda_{1}+20 \lambda_{4}\right.\right.\right. \\
& \left.+36 \lambda_{b}{ }^{2}+44 \lambda_{\tau}^{2}\right)-303 g_{2}{ }^{4} \\
& +4\left(25 \lambda_{1}\left(\lambda_{b}^{2}+3 \lambda_{\tau}^{2}\right)+108 \lambda_{1}^{2}\right. \\
& +4\left(6\left(2 \lambda_{3} \lambda_{4}+2 \lambda_{3}^{2}+\lambda_{4}^{2}\right)\right. \\
& \left.\left.\left.-3 \lambda_{5}{ }^{2}+4 \lambda_{b}{ }^{4}-12 \lambda_{\tau}{ }^{4}\right)\right)\right) \\
& +g_{1}{ }^{4}\left(-573 g_{2}{ }^{2}+651 \lambda_{1}+60\left(2 \lambda_{3}\right.\right. \\
& \left.\left.+\lambda_{4}+\lambda_{b}{ }^{2}-5 \lambda_{\tau}{ }^{2}\right)\right)-393 g_{1}{ }^{6} \\
& +3\left(-3 g_{2}{ }^{4}\left(17 \lambda_{1}+4\left(-10 \lambda_{3}\right.\right.\right. \\
& \left.\left.-5 \lambda_{4}+3 \lambda_{b}^{2}+\lambda_{\tau}^{2}\right)\right) \\
& +12 g_{2}^{2}\left(5 \lambda_{1}\left(3 \lambda_{b}^{2}+\lambda_{\tau}^{2}\right)\right. \\
& \left.+36 \lambda_{1}^{2}+4\left(2 \lambda_{3}+\lambda_{4}\right)^{2}\right)+291 g_{2}{ }^{6} \\
& -8\left(\lambda _ { 1 } \left(-80 g_{3}{ }^{2} \lambda_{b}{ }^{2}+20 \lambda_{3} \lambda_{4}+20 \lambda_{3}{ }^{2}\right.\right. \\
& \left.+12 \lambda_{4}{ }^{2}+14 \lambda_{5}{ }^{2}+3 \lambda_{b}{ }^{4}+\lambda_{\tau}{ }^{4}\right) \\
& +4\left(16 g_{3}{ }^{2} \lambda_{b}{ }^{4}+\lambda_{5}{ }^{2}\left(10 \lambda_{3}+11 \lambda_{4}\right)+8 \lambda_{3} \lambda_{4}{ }^{2}\right. \\
& \left.+6 \lambda_{3}{ }^{2} \lambda_{4}+4 \lambda_{3}{ }^{3}+3 \lambda_{4}{ }^{3}-5\left(3 \lambda_{b}{ }^{6}+\lambda_{\tau}{ }^{6}\right)\right)
\end{aligned}
$$

$$
\begin{aligned}
& \left.+24 \lambda_{1}^{2}\left(3 \lambda_{b}^{2}+\lambda_{\tau}^{2}\right)+78 \lambda_{1}^{3}\right) \\
& -24 \lambda_{t}^{2}\left(3 \lambda_{1} \lambda_{b}^{2}+8 \lambda_{3} \lambda_{4}+8 \lambda_{3}^{2}\right. \\
& \left.\left.\left.+4\left(\lambda_{4}^{2}+\lambda_{5}^{2}\right)-4 \lambda_{b}^{4}\right)\right)\right] .
\end{aligned}
$$$$
16 \pi^{2} \beta_{\lambda_{4}}=3 g_{1}{ }^{2}\left(g_{2}{ }^{2}-\lambda_{4}\right)-9 g_{2}{ }^{2} \lambda_{4}+2 \lambda_{4}\left(\lambda_{1}+\lambda_{2}\right.
$$$$
\left.+4 \lambda_{3}+2 \lambda_{4}+3 \lambda_{b}^{2}+\lambda_{\tau}^{2}\right)
$$$$
+6 \lambda_{t}{ }^{2}\left(\lambda_{4}+2 \lambda_{b}{ }^{2}\right)+8 \lambda_{5}^{2}
$$$$
+\frac{1}{384 \pi^{2}}\left[2 g _ { 1 } ^ { 2 } \left(3 g _ { 2 } ^ { 2 } \left(20 \lambda_{1}+20 \lambda_{2}+8 \lambda_{3}\right.\right.\right.
$$$$
\left.+51 \lambda_{4}+36 \lambda_{b}^{2}+44 \lambda_{\tau}^{2}+84 \lambda_{t}^{2}\right)
$$$$
-168 g_{2}{ }^{4}+\lambda_{4}\left(48 \lambda_{1}+48 \lambda_{2}+48 \lambda_{3}\right.
$$$$
\left.+96 \lambda_{4}+25 \lambda_{b}^{2}+75 \lambda_{\tau}^{2}\right)
$$$$
\left.+\lambda_{t}{ }^{2}\left(85 \lambda_{4}+16 \lambda_{b}{ }^{2}\right)+192 \lambda_{5}^{2}\right)
$$$$
+g_{1}{ }^{4}\left(471 \lambda_{4}-876 g_{2}^{2}\right)
$$$$
+3\left(6 g _ { 2 } ^ { 2 } \left(\lambda _ { 4 } \left(48 \lambda_{3}+24 \lambda_{4}+5\left(3 \lambda_{b}^{2}+\lambda_{\tau}^{2}\right.\right.\right.\right.
$$$$
\left.\left.\left.+3 \lambda_{t}{ }^{2}\right)\right)+72 \lambda_{5}{ }^{2}\right)-231 g_{2}{ }^{4} \lambda_{4}
$$$$
+4\left(-8 \lambda_{b}{ }^{2}\left(-10 g_{3}{ }^{2} \lambda_{4}+3 \lambda_{4}\left(\lambda_{1}\right.\right.\right.
$$$$
\left.\left.+2 \lambda_{3}+\lambda_{4}\right)+6 \lambda_{5}^{2}\right)
$$$$
-2 \lambda_{t}{ }^{2}\left(3 \left(4 \lambda_{4}\left(\lambda_{2}+2 \lambda_{3}+\lambda_{4}\right)+\lambda_{b}^{2}\left(8 \lambda_{3}\right.\right.\right.
$$$$
\left.\left.+11 \lambda_{4}\right)+8 \lambda_{5}^{2}+8 \lambda_{b}^{4}\right)
$$$$
\left.-8 g_{3}^{2}\left(5 \lambda_{4}+8 \lambda_{b}{ }^{2}\right)\right)-4 \lambda_{5}^{2}\left(1 2 \left(\lambda_{1}+\lambda_{2}\right.\right.
$$$$
\left.\left.+2 \lambda_{3}\right)+13 \lambda_{4}\right)
$$$$
-2 \lambda_{4}\left(20 \lambda_{1}\left(2 \lambda_{3}+\lambda_{4}\right)+7 \lambda_{1}^{2}+20 \lambda_{2}\left(2 \lambda_{3}+\lambda_{4}\right)\right.
$$$$
\left.+7 \lambda_{2}^{2}+28 \lambda_{3}\left(\lambda_{3}+\lambda_{4}\right)\right)
$$$$
-8 \lambda_{\tau}^{2}\left(\lambda_{4}\left(\lambda_{1}+2 \lambda_{3}+\lambda_{4}\right)+2 \lambda_{5}^{2}\right)
$$$$
\left.\left.\left.-3 \lambda_{t}{ }^{4}\left(9 \lambda_{4}+16 \lambda_{b}{ }^{2}\right)-27 \lambda_{4} \lambda_{b}{ }^{4}-9 \lambda_{4} \lambda_{\tau}{ }^{4}\right)\right)\right] \text {. }
$$

$$
\begin{aligned}
16 \pi^{2} \beta_{\lambda_{5}}= & \lambda_{5}\left(-3 g_{1}{ }^{2}-9 g_{2}{ }^{2}+2\left(\lambda_{1}+\lambda_{2}+4 \lambda_{3}\right.\right. \\
& \left.\left.+6 \lambda_{4}+3 \lambda_{b}{ }^{2}+\lambda_{\tau}{ }^{2}+3 \lambda_{t}{ }^{2}\right)\right) \\
& +\frac{1}{384 \pi^{2}}\left[\lambda _ { 5 } \left(2 g _ { 1 } { } ^ { 2 } \left(57 g_{2}{ }^{2}-24 \lambda_{1}-24 \lambda_{2}\right.\right.\right. \\
& \left.+192 \lambda_{3}+288 \lambda_{4}+25 \lambda_{b}{ }^{2}+75 \lambda_{\tau}{ }^{2}+85 \lambda_{t}{ }^{2}\right) \\
& +471 g_{1}{ }^{4}+3\left(6 g _ { 2 } { } ^ { 2 } \left(48 \lambda_{3}+96 \lambda_{4}+5\left(3 \lambda_{b}{ }^{2}\right.\right.\right.
\end{aligned}
$$




$$
\begin{aligned}
& \left.\left.+\lambda_{\tau}{ }^{2}+3 \lambda_{t}^{2}\right)\right)-231 g_{2}{ }^{2} \\
& +4\left(-8 \lambda_{b}^{2}\left(-10 g_{3}^{2}+3 \lambda_{1}+6 \lambda_{3}+9 \lambda_{4}\right)\right. \\
& -2 \lambda_{t}{ }^{2}\left(-40 g_{3}{ }^{2}+12\left(\lambda_{2}+2 \lambda_{3}+3 \lambda_{4}\right)\right. \\
& \left.+33 \lambda_{b}{ }^{2}\right)-8 \lambda_{4}\left(11\left(\lambda_{1}+\lambda_{2}\right)+19 \lambda_{3}\right) \\
& -8 \lambda_{3}\left(10\left(\lambda_{1}+\lambda_{2}\right)+7 \lambda_{3}\right)-8 \lambda_{\tau}^{2}\left(\lambda_{1}+2 \lambda_{3}\right. \\
& \left.\left.+3 \lambda_{4}\right)-64 \lambda_{4}{ }^{2}-3 \lambda_{b}{ }^{4}-\lambda_{\tau}{ }^{4}-3 \lambda_{t}{ }^{4}\right) \\
& \left.\left.\left.-56\left(\lambda_{1}{ }^{2}+\lambda_{2}{ }^{2}\right)+48 \lambda_{5}{ }^{2}\right)\right)\right] .
\end{aligned}
$$

The $\beta$ functions for the Yukawa couplings $\lambda_{t}, \lambda_{b}$ and $\lambda_{\tau}$ are given by:

$$
\begin{aligned}
& 16 \pi^{2} \beta_{\lambda_{t}}=\frac{1}{12} \lambda_{t}\left(-17 g_{1}(t)^{2}-27 g_{2}(t)^{2}\right. \\
& \left.+6\left(-16 g_{3}(t)^{2}+\lambda_{b}^{2}+9 \lambda_{t}^{2}\right)\right) \\
& -\frac{1}{6912 \pi^{2}}\left[\lambda _ { t } \left(-2534 g_{1}{ }^{4}+3 g_{1}^{2}\left(108 g_{2}{ }^{2}\right.\right.\right. \\
& \left.-304 g_{3}^{2}+41 \lambda_{b}^{2}-1179 \lambda_{t}^{2}\right) \\
& +9\left(252 g_{2}{ }^{4}-9 g_{2}{ }^{2}\left(48 g_{3}{ }^{2}+11 \lambda_{b}{ }^{2}+75 \lambda_{t}{ }^{2}\right)\right. \\
& +4\left(1296 g_{3}{ }^{4}-16 g_{3}{ }^{2}\left(4 \lambda_{b}{ }^{2}+27 \lambda_{t}{ }^{2}\right)\right. \\
& -3\left(6 \lambda_{2}^{2}-24 \lambda_{2} \lambda_{t}^{2}+4 \lambda_{3}^{2}+4 \lambda_{3} \lambda_{4}\right. \\
& -8 \lambda_{3} \lambda_{b}{ }^{2}+4 \lambda_{4}{ }^{2}+8 \lambda_{4} \lambda_{b}{ }^{2} \\
& +6 \lambda_{5}{ }^{2}-10 \lambda_{b}{ }^{4}-3 \lambda_{b}{ }^{2} \lambda_{\tau}{ }^{2} \\
& \left.\left.\left.\left.\left.-10 \lambda_{b}{ }^{2} \lambda_{t}{ }^{2}-48 \lambda_{t}{ }^{4}\right)\right)\right)\right)\right] \\
& \left.\left.+9 \lambda_{b}^{2}+2 \lambda_{\tau}^{2}+\lambda_{t}^{2}\right)\right) \\
& -\frac{1}{6912 \pi^{2}}\left[\lambda _ { b } \left(226 g_{1}{ }^{4}+3 g_{1}{ }^{2}\left(324 g_{2}{ }^{2}\right.\right.\right. \\
& \left.-496 g_{3}{ }^{2}-711 \lambda_{b}{ }^{2}-450 \lambda_{\tau}{ }^{2}+53 \lambda_{t}{ }^{2}\right) \\
& +9\left(252 g_{2}{ }^{4}-9 g_{2}{ }^{2}\left(48 g_{3}{ }^{2}+75 \lambda_{b}{ }^{2}\right.\right. \\
& \left.+10 \lambda_{\tau}^{2}+11 \lambda_{t}^{2}\right) \\
& +4\left(1296 g_{3}{ }^{4}-16 g_{3}{ }^{2}\left(27 \lambda_{b}{ }^{2}+4 \lambda_{t}{ }^{2}\right)\right. \\
& -3\left(6 \lambda_{1}^{2}-24 \lambda_{1} \lambda_{b}^{2}+4 \lambda_{3}^{2}+4 \lambda_{3} \lambda_{4}\right. \\
& -8 \lambda_{3} \lambda_{t}^{2}+4 \lambda_{4}^{2}+8 \lambda_{4} \lambda_{t}^{2}+6 \lambda_{5}^{2}-48 \lambda_{b}{ }^{4} \\
& \left.\left.\left.\left.\left.-9 \lambda_{b}{ }^{2} \lambda_{\tau}{ }^{2}-10 \lambda_{b}{ }^{2} \lambda_{t}{ }^{2}-9 \lambda_{\tau}{ }^{4}-10 \lambda_{t}{ }^{4}\right)\right)\right)\right)\right]
\end{aligned}
$$

$$
16 \pi^{2} \beta_{\lambda_{\tau}}=\frac{1}{4} \lambda_{\tau}\left(-15 g_{1}{ }^{2}-9 g_{2}{ }^{2}+12 \lambda_{b}{ }^{2}+10 \lambda_{\tau}{ }^{2}\right)
$$

$$
\begin{aligned}
& -\frac{1}{768 \pi^{2}}\left[\lambda _ { \tau } \left(-966 g_{1}{ }^{4}-g_{1}{ }^{2}\left(108 g_{2}{ }^{2}\right.\right.\right. \\
& \left.+50 \lambda_{b}{ }^{2}+537 \lambda_{\tau}{ }^{2}\right) \\
& +3\left(84 g_{2}{ }^{4}-15 g_{2}{ }^{2}\left(6 \lambda_{b}{ }^{2}+11 \lambda_{\tau}{ }^{2}\right)\right. \\
& -4\left(80 g_{3}{ }^{2} \lambda_{b}{ }^{2}+6 \lambda_{1}{ }^{2}-24 \lambda_{1} \lambda_{\tau}{ }^{2}\right. \\
& +4 \lambda_{3}{ }^{2}+4 \lambda_{3} \lambda_{4}+4 \lambda_{4}{ }^{2}+6 \lambda_{5}{ }^{2}-27 \lambda_{b}{ }^{4} \\
& \left.\left.\left.\left.-27 \lambda_{b}{ }^{2} \lambda_{\tau}{ }^{2}-9 \lambda_{b}{ }^{2} \lambda_{t}{ }^{2}-12 \lambda_{\tau}{ }^{4}\right)\right)\right)\right]
\end{aligned}
$$

\section{References}

1. ATLAS collaboration, G. Aad et al., Observation of a new particle in the search for the Standard Model Higgs boson with the ATLAS detector at the LHC. Phys. Lett. B 716, 1 (2012). arXiv: 1207.7214

2. CMS collaboration, S. Chatrchyan et al., Observation of a new boson at a mass of $125 \mathrm{GeV}$ with the CMS experiment at the LHC. Phys. Lett. B 716, 30 (2012). arXiv:1207.7235

3. J. Elias-Miro, J.R. Espinosa, G.F. Giudice, G. Isidori, A. Riotto, A. Strumia, Higgs mass implications on the stability of the electroweak vacuum. Phys. Lett. B 709, 222 (2012). arXiv:1112.3022

4. V. Branchina, E. Messina, Stability, Higgs boson mass and new physics. Phys. Rev. Lett. 111, 241801 (2013). arXiv:1307.5193

5. D. Buttazzo, G. Degrassi, P.P. Giardino, G.F. Giudice, F. Sala, A. Salvio et al., Investigating the near-criticality of the Higgs boson. JHEP 12, 089 (2013). arXiv:1307.3536

6. M. Shaposhnikov, C. Wetterich, Asymptotic safety of gravity and the Higgs boson mass. Phys. Lett. B 683, 196 (2010). arXiv:0912.0208

7. M. Holthausen, K.S. Lim, M. Lindner, Planck scale boundary conditions and the Higgs mass. JHEP 1202, 037 (2012). arXiv: 1112.2415

8. S. Weinberg, Ultraviolet divergencies in theories of quantum gravitation, in General Relativity: An Einstein Centenary Survey, ed. by S. Hawking, W. Israel (Cambridge University Press, Cambridge, 1979), pp. 790-831

9. C. Wetterich, Exact evolution equation for the effective potential. Phys. Lett. B 301, 90 (1993)

10. R. Percacci, A short introduction to asymptotic safety. arXiv: 1110.6389

11. A.D. Bond, G. Hiller, K. Kowalska, D.F. Litim, Directions for model building from asymptotic safety. JHEP 08, 004 (2017). arXiv: 1702.01727

12. G.M. Pelaggi, A.D. Plascencia, A. Salvio, F. Sannino, J. Smirnov, A. Strumia, Asymptotically safe standard model extensions? arXiv: 1708.00437

13. A.D. Bond, D.F. Litim, More asymptotic safety guaranteed. arXiv: 1707.04217

14. A.D. Bond, D.F. Litim, Price of asymptotic safety. arXiv: 1801.08527

15. D. Barducci, M. Fabbrichesi, C.M. Nieto, R. Percacci, V. Skrinjar, In search of a UV completion of the Standard Model-378.000 models that don't work. arXiv:1807.05584

16. R. Mann, J. Meffe, F. Sannino, T. Steele, Z.-W. Wang, C. Zhang, Asymptotically safe standard model via vectorlike fermions. Phys. Rev. Lett. 119, 261802 (2017). arXiv: 1707.02942

17. A.D. Bond, D.F. Litim, Theorems for asymptotic safety of gauge theories. arXiv:1608.00519 
18. A.V. Bednyakov, B.A. Kniehl, A.F. Pikelner, O.L. Veretin, Stability of the electroweak vacuum: gauge independence and advanced precision. Phys. Rev. Lett. 115, 201802 (2015). arXiv: 1507.08833

19. N. Turok, J. Zadrozny, Electroweak baryogenesis in the two doublet model. Nucl. Phys. B 358, 471 (1991)

20. M. Joyce, T. Prokopec, N. Turok, Nonlocal electroweak baryogenesis. Part 2: the classical regime. Phys. Rev. D 53, 2958 (1996). arXiv:hep-ph/9410282

21. L. Fromme, S.J. Huber, M. Seniuch, Baryogenesis in the two-Higgs doublet model. JHEP 11, 038 (2006). arXiv:hep-ph/0605242

22. N.G. Deshpande, E. Ma, Pattern of symmetry breaking with two Higgs doublets. Phys. Rev. D 18, 2574 (1978)

23. R. Barbieri, L.J. Hall, V.S. Rychkov, Improved naturalness with a heavy Higgs: an alternative road to LHC physics. Phys. Rev. D 74, 015007 (2006). arXiv:hep-ph/0603188

24. L. Lopez Honorez, E. Nezri, J.F. Oliver, M.H.G. Tytgat, The inert doublet model: an archetype for dark matter. JCAP 0702, 028 (2007). arXiv:hep-ph/0612275

25. P.M. Ferreira, R. Santos, M. Sher, J.P. Silva, Implications of the LHC two-photon signal for two-Higgs-doublet models. Phys. Rev. D 85, 077703 (2012). arXiv: 1112.3277

26. C.-Y. Chen, S. Dawson, Exploring two Higgs doublet models through Higgs production. Phys. Rev. D 87, 055016 (2013). arXiv: 1301.0309

27. P. Bechtle, S. Heinemeyer, O. Stål, T. Stefaniak, G. Weiglein, Higgs Signals: confronting arbitrary Higgs sectors with measurements at the Tevatron and the LHC. Eur. Phys. J. C 74, 2711 (2014). arXiv: 1305.1933

28. T.D. Lee, A theory of spontaneous T violation. Phys. Rev. D 8, 1226 (1973)

29. G.C. Branco, P.M. Ferreira, L. Lavoura, M.N. Rebelo, M. Sher, J.P. Silva, Theory and phenomenology of two-Higgs-doublet models. Phys. Rep. 516, 1 (2012). arXiv: 1106.0034

30. E.A. Paschos, Diagonal neutral currents. Phys. Rev. D 15, 1966 (1977)

31. S.L. Glashow, S. Weinberg, Natural conservation laws for neutral currents. Phys. Rev. D 15, 1958 (1977)

32. M. Aoki, S. Kanemura, K. Tsumura, K. Yagyu, Models of Yukawa interaction in the two Higgs doublet model, and their collider phenomenology. Phys. Rev. D 80, 015017 (2009). arXiv:0902.4665

33. V. Branchina, F. Contino, P.M. Ferreira, Electroweak vacuum lifetime in two Higgs doublet models. arXiv:1807.10802

34. P.M. Ferreira, R. Santos, A. Barroso, Stability of the tree-level vacuum in two Higgs doublet models against charge or $\mathrm{CP}$ spontaneous violation. Phys. Lett. B 603, 219 (2004). arXiv:hep-ph/0406231

35. A. Barroso, P.M. Ferreira, R. Santos, Charge and CP symmetry breaking in two Higgs doublet models. Phys. Lett. B 632, 684 (2006). arXiv:hep-ph/0507224

36. A. Barroso, P.M. Ferreira, R. Santos, Neutral minima in two-Higgs doublet models. Phys. Lett. B 652, 181 (2007). arXiv:hep-ph/0702098

37. A. Barroso, P.M. Ferreira, I.P. Ivanov, R. Santos, Metastability bounds on the two Higgs doublet model. JHEP 06, 045 (2013). arXiv: 1303.5098

38. Barroso, A., Ferreira, P.M., Santos, R., Sher, M., Silva, J.P.: 2HDM at the LHC - the story so far, in Proceedings, 1st Toyama International Workshop on Higgs as a Probe of New Physics 2013 (HPNP2013): Toyama, Japan, February 13-16, 2013 (2013). arXiv: 1304.5225

39. B. Coleppa, F. Kling, S. Su, Constraining type II $2 \mathrm{HDM}$ in light of LHC Higgs searches. JHEP 01, 161 (2014). arXiv:1305.0002

40. CMS collaboration, A.M. Sirunyan, et al., Combined measurements of Higgs boson couplings in proton-proton collisions at $\sqrt{s}=13$ TeV. arXiv: 1809.10733

41. O. Eberhardt, Two-Higgs-doublet model fits with HEPfit, in 2017 European Physical Society Conference on High Energy
Physics (EPS-HEP 2017) Venice, Italy, July 5-12, 2017 (2017). arXiv: 1709.09414

42. D. Chowdhury, O. Eberhardt, Update of global two-Higgs-doublet model fits. arXiv:1711.02095

43. G.C. Dorsch, S.J. Huber, K. Mimasu, J.M. No, Hierarchical versus degenerate 2HDM: the LHC run 1 legacy at the onset of run 2. Phys. Rev. D 93, 115033 (2016). arXiv:1601.04545

44. H.S. Cheon, S.K. Kang, Constraining parameter space in type-II two-Higgs doublet model in light of a $126 \mathrm{GeV}$ Higgs boson. JHEP 09, 085 (2013). arXiv: 1207.1083

45. O. Eberhardt, U. Nierste, M. Wiebusch, Status of the two-Higgsdoublet model of type II. JHEP 07, 118 (2013). arXiv:1305.1649

46. D. Chowdhury, O. Eberhardt, Global fits of the two-loop renormalized two-Higgs-doublet model with soft $Z_{2}$ breaking. JHEP 11, 052 (2015). arXiv: 1503.08216

47. N. Chakrabarty, U.K. Dey, B. Mukhopadhyaya, High-scale validity of a two-Higgs doublet scenario: a study including LHC data. JHEP 12, 166 (2014). arXiv: 1407.2145

48. B.W. Lee, C. Quigg, H.B. Thacker, The strength of weak interactions at very high-energies and the Higgs boson mass. Phys. Rev. Lett. 38, 883 (1977)

49. B. Grinstein, C.W. Murphy, P. Uttayarat, One-loop corrections to the perturbative unitarity bounds in the CP-conserving two-Higgs doublet model with a softly broken $\mathrm{Z}_{2}$ symmetry. JHEP 06, 070 (2016). arXiv:1512.04567

50. ATLAS collaboration, T.A. collaboration, Updated coupling measurements of the Higgs boson with the ATLAS detector using up to $25 \mathrm{fb}^{-1}$ of proton-proton collision data

51. CMS collaboration, C. Collaboration, Precise determination of the mass of the Higgs boson and studies of the compatibility of its couplings with the standard model

52. ATLAS collaboration, T.A. collaboration, Combined measurement of differential and inclusive total cross sections in the $H \rightarrow \gamma \gamma$ and the $H \rightarrow Z Z^{*} \rightarrow 4 \ell$ decay channels at $\sqrt{s}=13 \mathrm{TeV}$ with the ATLAS detector

53. CMS collaboration, C. Collaboration, Combined measurements of the Higgs boson's couplings at $\sqrt{s}=13 \mathrm{TeV}$

54. T. Enomoto, R. Watanabe, Flavor constraints on the two Higgs doublet models of $Z_{2}$ symmetric and aligned types. arXiv:1511.05066

55. M. Misiak, M. Steinhauser, Weak radiative decays of the B meson and bounds on $M_{H^{ \pm}}$in the two-Higgs-doublet model. Eur. Phys. J. C 77, 201 (2017). arXiv:1702.04571

56. J. Haller, A. Hoecker, R. Kogler, K. Mönig, T. Peiffer, J. Stelzer, Update of the global electroweak fit and constraints on two-Higgsdoublet models. Eur. Phys. J. C 78, 675 (2018). arXiv:1803.01853

57. J.F. Gunion, H.E. Haber, The CP conserving two Higgs doublet model: the approach to the decoupling limit. Phys. Rev. D 67, 075019 (2003). arXiv:hep-ph/0207010

58. D. Das, I. Saha, Search for a stable alignment limit in two-Higgs-doublet models. Phys. Rev. D 91, 095024 (2015). arXiv:1503.02135

59. M. Carena, I. Low, N.R. Shah, C.E.M. Wagner, Impersonating the standard model Higgs boson: alignment without decoupling. JHEP 04, 015 (2014). arXiv: 1310.2248

60. P.S. Bhupal Dev, A. Pilaftsis, Maximally symmetric two Higgs doublet model with natural standard model alignment. JHEP 12, 024 (2014). arXiv:1408.3405

61. F. Staub, SARAH. arXiv:0806.0538

62. F. Staub, SARAH 4: a tool for (not only SUSY) model builders. Comput. Phys. Commun. 185, 1773 (2014). arXiv:1309.7223

63. P.J. Mohr, D.B. Newell, B.N. Taylor, CODATA recommended values of the fundamental physical constants: 2014. Rev. Mod. Phys. 88, 035009 (2016). arXiv:1507.07956

64. Particle Data Group collaboration, K.A. Olive et al., Review of particle physics. Chin. Phys. C 38, 090001 (2014) 
65. A. Falkowski, C. Gross, O. Lebedev, A second Higgs from the Higgs portal. JHEP 05, 057 (2015). arXiv:1502.01361

66. P.M. Ferreira, R. Santos, M. Sher, J.P. Silva, Could the LHC two-photon signal correspond to the heavier scalar in two-Higgsdoublet models? Phys. Rev. D 85, 035020 (2012). arXiv:1201.0019

67. G. Isidori, G. Ridolfi, A. Strumia, On the metastability of the standard model vacuum. Nucl. Phys. B 609, 387 (2001). arXiv:hep-ph/0104016

68. G. Degrassi, S. Di Vita, J. Elias-Miró, J.R. Espinosa, G.F. Giudice, G. Isidori, A. Strumia, Higgs mass and vacuum stability in the Standard Model at NNLO. High Energy Phys. Phenomenol. arXiv:1205.6497 [hep-ph]

69. N. Chakrabarty, B. Mukhopadhyaya, High-scale validity of a two Higgs doublet scenario: metastability included. Eur. Phys. J. C 77, 153 (2017). arXiv: 1603.05883
70. X.-D. Cheng, Y.-D. Yang, X.-B. Yuan, Revisiting $B_{s} \rightarrow \mu^{+} \mu^{-}$in the two-Higgs doublet models with $Z_{2}$ symmetry. Eur. Phys. J. C 76, 151 (2016). arXiv: 1511.01829

71. J. McDowall, D.J. Miller, High scale boundary conditions in models with two Higgs doublets. arXiv: 1810.04518

72. M.E. Machacek, M.T. Vaughn, Two loop renormalization group equations in a general quantum field theory. 1. Wave function renormalization. Nucl. Phys. B B222, 83 (1983)

73. M.E. Machacek, M.T. Vaughn, Two loop renormalization group equations in a general quantum field theory. 2. Yukawa couplings. Nucl. Phys. B B236, 221 (1984)

74. M.E. Machacek, M.T. Vaughn, Two loop renormalization group equations in a general quantum field theory. 3. Scalar quartic couplings. Nucl. Phys. B B249, 70 (1985) 